SINGLE-MODE FIBER,

VELOCITY INTERFEROMETRY

K. G. Krauter, G. F. Jacobson, J. R. Patterson, J.

H. Nguyen, W. P. Ambrose

December 16, 2010

Review of Scientific Instruments 
This document was prepared as an account of work sponsored by an agency of the United States government. Neither the United States government nor Lawrence Livermore National Security, LLC, nor any of their employees makes any warranty, expressed or implied, or assumes any legal liability or responsibility for the accuracy, completeness, or usefulness of any information, apparatus, product, or process disclosed, or represents that its use would not infringe privately owned rights. Reference herein to any specific commercial product, process, or service by trade name, trademark, manufacturer, or otherwise does not necessarily constitute or imply its endorsement, recommendation, or favoring by the United States government or Lawrence Livermore National Security, LLC. The views and opinions of authors expressed herein do not necessarily state or reflect those of the United States government or Lawrence Livermore National Security, LLC, and shall not be used for advertising or product endorsement purposes. 


\title{
SINGLE-MODE FIBER, VELOCITY INTERFEROMETRY
}

K. G. Krauter, G. F. Jacobson, J. R. Patterson, J. H. Nguyen, and W. P. Ambrose Lawrence Livermore National Laboratory, 7000 East Ave, Livermore CA 94551

REVISED NOVEMBER 2010

\begin{abstract}
In this paper we describe a velocity interferometer system (a VISAR) based entirely on single-mode fiber optics. This paper includes a description of principles used in developing the single-mode velocity interferometry system (SMV). The SMV design is based on polarizationinsensitive components. Polarization adjusters are included to eliminate the effects of residual birefringence and polarization dependent losses in the interferometers. Characterization measurements and calibration methods needed for data analysis and a method of data analysis are described. Calibration is performed directly using tunable lasers.

During development, we demonstrated its operation using exploding-foil bridge-wire fliers up to $200 \mathrm{~m} / \mathrm{s}$. In a final test, we demonstrated the SMV in a gas gun experiment up to 1.2 $\mathrm{km} / \mathrm{sec}$. As a basis for comparison in the gas gun experiment, we used another velocimetry technique that is also based on single mode fiber optics: photonic Doppler velocimetry (PDV). For the gas gun experiment, we split the light returned from a single target spot, and performed a direct comparison of the homodyne (SMV) and heterodyne (PDV) techniques concurrently. The two techniques had a negligible mean difference and a $1.5 \%$ standard deviation in the 1 dimensional shock zone.

Within one interferometer delay time after a sudden Doppler shift, an SMV unencumbered by multimode-fiber dispersion exhibits two color beats. These beats have the same period as PDV beats -- this interference occurs between the "recently" shifted and "formerly un-shifted" paths within the interferometer. We believe that recognizing this identity between homodyne and heterodyne beats is novel in the shock-physics field. SMV includes the conveniences of optical fiber, while removing the time resolution limitations associated with multimode delivery fiber.
\end{abstract}




\section{INTRODUCTION}

High pressure shock-wave experiments produce rapid changes in material properties in response to high load rates. Material-strength changes and phase transitions occur on nanosecond time scales in impact experiments - on these short time scales, the response of materials can be remarkably different than expected when compared to slower loading. The different macroscopic dynamics observed under high load rates has inspired theories that attempt to predict the underlying rates of microscopic mechanisms responsible for the observed differences in behavior (for recent reviews, see [1-5]).

Macroscopic dynamics are observed by measuring material-surface velocities. Mass velocities up to a few $\mathrm{km} / \mathrm{s}$ are generated in single-shock, propellant-driven, flier-plate impact experiments [1-3]. Higher velocities have been generated in pulsed-laser and pulsed-plasma driven experiments up to $19 \mathrm{~km} / \mathrm{s}$ [6], $30 \mathrm{~km} / \mathrm{s}$ [4], and $\sim 100 \mathrm{~km} / \mathrm{s}$ [5]. Improving the temporal and velocity resolution of high-velocity measurements continues to be of interest as it will lead to improved data needed to better understand high strain rate processes.

In this paper we describe a VISAR ("velocity interferometer system for any reflector" [7,8]) based entirely on single-mode fiber optics. We refer to the technique as single-mode VISAR (SMV). The use of single mode fiber incorporates the convenient aspects of optical fiber for light delivery, while improving the temporal resolution compared to VISARs that use multimode fiber.

To place this work in context, we review previous developments in velocimetries with relevance to the present work. In particular, we refer to developments involving: the application of homodyne and heterodyne detection to velocimetry; VISAR and PDV (photonic Doppler velocimetry); removing extraneous amplitude modulation; the historical introduction of fiber optics with a positive side effect of enabling the scrambling of polarization modulation; and various performance limits on time resolution and velocity resolution introduced by each development.

Independent of instrumentation, optical velocimeters utilize a common principle; the optical frequency of a laser source is Doppler shifted upon reflection from a moving surface. The 
frequency shift is proportional to the reflecting-surface velocity. Methods of detecting the Doppler frequency shift can be divided generally into two categories: heterodyned detection [9$12]$ and homodyned detection $[13,14]$. It should not be surprising that time resolution and velocity sensitivity are inversely related in both heterodyne and homodyne detection systems. However, as we illustrate in the next few paragraphs, there are differences in the ways in which heterodyne and homodyne systems link the limits on velocity sensitivity and time resolution to other parameters such as velocity and available technologies.

In a homodyne system frequency shifts are inferred from interference-phase shifts measured as power oscillations at the output of an interferometer $[13,15,16]$. VISAR is an example of a homodyne detection system. In a VISAR, the Doppler shifted light returned from the target is injected into an interferometer with unequal interference path lengths. The light is split, propagates in the unequal paths, is recombined and detected. A VISAR's signal contains interference terms similar to $\cos (2 \pi V(t) / V P F)$, where $\mathrm{V}(\mathrm{t})$ is the velocity and $\mathrm{VPF}$ is a velocity per fringe constant. Since VISAR converts increasing velocity into cyclically varying signals (the range of power and signal amplitude are bounded), the range of velocities that VISAR can measure is not limited by recording technology and is practically unlimited.

When a VISAR-like homodyne system is used to measure processes occurring under extreme conditions, signals similar to $\cos (2 \pi V(t) / V P F)$ need to be distinguished from other sources of amplitude modulation such as bright flashes of light or changes in reflectance. The development of the push-pull VISAR [16] allowed removal of extraneous sources of light. Push-pull VISAR incorporates four detector outputs that allow extraneous light to be removed by subtraction of pairs of interference signals (with $180^{\circ}$ phase). Subsequent division by a similar term with a different phase is then used to remove residual power variation introduce by surface-reflectance changes (this last set of statements can be understood more fully by following the development in appendix B). The SMV described in this paper incorporates removal of extraneous amplitude modulation similarly to a push-pull VISAR.

Developments that led up to the push-pull VISAR were made more than twenty years ago using free space optics between the experiment and the interferometer [7, 13, 16-18]. More recently optical fiber was introduced as a means of light delivery from laser to target, and target to interferometer [19], while continuing to use free space optics in the interferometer. The use of 
optical fiber removes strict alignment requirements between target and the input to an interferometer. The use of multimode fiber also satisfies a second problem for the push pull VISAR that is related to polarization changes.

Rapidly-changing induced-birefringence effects are known to occur in shock physics experiments [20]. Since push-pull VISAR is based on polarizing optics, changes in the polarization state of the light can also introduce amplitude modulation. One reliable method to avoid additional amplitude modulation due to changes in polarization is to intentionally depolarize or rather scramble the distribution of polarization angles of either the incident or return light [21]. The introduction of multimode fiber for light delivery in combination with the push-pull VISAR had provided a readily available and simple means to depolarize the return light. The single mode fiber based instrument we describe below uses a different approach - we avoid the use of optical elements with a strong transmission dependence on polarization in order to avoid additional amplitude modulation introduced by target-polarization changes.

Although multimode optical fiber eliminates the need for free space alignment and enables polarization scrambling for the push-pull VISAR, the use of multimode fiber for transmission has one drawback. Multimode fiber introduces a performance limitation on the time resolution due to the different propagation times for different paths (or modes) in the optical fiber. Multimode-time dispersion can dominate the instrument's response time after $20 \mathrm{~m}$ of fiber, where typical rise times and bandwidths of graded index multimode fiber can be $\sim 0.5 \mathrm{~ns}$ and $\sim$ $0.6 \mathrm{GHz}$.

An alternative to homodyne detection is heterodyne detection. The use of heterodyne detection in velocimetry (also known as displacement interferometry) was first demonstrated 40 years ago [9]. In a heterodyned system the Doppler shifted light is combined with a similar amount of un-shifted laser light. The frequency difference (or frequency shift) between the unshifted reference frequency and the Doppler shifted frequency is manifested as a beat frequency in the detected signal. In 1965, velocities in heterodyne detection techniques were limited to $100 \mathrm{~m} / \mathrm{s}$ because of the available bandwidth of detectors and recording electronics. Because of the inherently unlimited velocity in VISAR systems, heterodyne detection apparently was abandoned in favor of VISAR developments at an early date [18]. 
More recent technological advances have caused a rediscovery and revival of heterodyne detection. Photonic Doppler Velocimetry (PDV) is a successful high-speed velocimetry technique that was developed around heterodyne detection using near-infrared single-mode fiber technologies [10-12, 22]. Since PDV uses optical fiber, PDV also avoids the constraints of free space optics. Since it uses single mode fiber, the optical portion of the system has high temporal resolution. Since the velocity is encoded at high modulation frequencies, PDV appears to be relatively immune to many of the low-frequency amplitude modulation effects that had led to the use of push-pull VISAR in combination with multimode fiber.

Since PDV is based entirely on commercially available single-mode fiber components and detectors, the photonic portion of the instrumentation can have a bandwidth exceeding $20 \mathrm{GHz}$. Commercially available single-shot digital recorders with long record lengths (milliseconds) are now available with bandwidth up to $20 \mathrm{GHz}$ and time resolution down to $22 \mathrm{ps.} 20 \mathrm{GHz}$ corresponds to a velocity of $15 \mathrm{~km} / \mathrm{sec}$ when using a wavelength of $1550 \mathrm{~nm}$. For velocities below $15 \mathrm{~km} / \mathrm{sec}$, the only drawback to PDV appears to be the high cost of detectors and recording electronics when a bandwidth as high as $20 \mathrm{GHz}$ is required.

VISAR-interferometer designs based on single mode fiber have been attempted [23-25]. Fiber-based VISARs have not yet addressed problems that were solved in the development of the free space push-pull VISAR designs. A lack of polarization compensation, a lack of compensation for extraneous light effects or target-reflectance variation, and a method of accurate calibration have not been developed for fiber-based VISARs. Velocities above $120 \mathrm{~m} / \mathrm{s}$ [23] have not been reported for VISARs based on single mode fiber.

In this paper, we describe a novel optical velocimeter combining positive attributes of technologies from PDV and VISAR: PDV removes a practical time-resolution limit by replacing multimode fiber with single mode fiber, while VISARs have the advantage that the velocity is not limited by costly electronics. The velocity interferometry system described in this paper incorporates concepts from VISARs, is based entirely on single-mode fiber components, near infrared lasers, and fast photodetectors that are similar to components in use in PDV.

In this paper we explain the principles used in the SMV apparatus, demonstrate its operation using exploding-foil bridge-wire fliers, and compare the accuracy of the SMV with PDV in a gas gun experiment at $1 \mathrm{~km} / \mathrm{sec}$. For the gas gun experiment, we compare the results obtained using 
both homodyne (SMV) and heterodyne (PDV) detection for the same target spot. As described in Appendix A, rather than intentionally scrambling the polarization to remove sensitivity to polarization modulation produced by the target (as used in push-pull VISAR with multimode fiber), for SMV we incorporate only unpolarized components and build in compensation to remove residual birefringence and polarization dependent losses. The method of data analysis has similarities to the analysis of push pull VISARs [16,21], but the analysis requires modifications for SMV that are described in Appendix B. SMV includes the conveniences of optical fiber, removes the time resolution limitations associated with multimode delivery fiber, while removing the need to use costly digital recording electronics to reach high velocities.

\section{SMV APPARATUS DESIGN}

Figure 1 shows the design for the SMV. Between the laser and the detectors (D), all optical paths utilize single-mode optical fiber components. Components in the upper portion of the diagram (above the 50/50 coupler labeled i) are used to route light to and from the target surface. The SMV interferometers, detectors and recorders are below the splitter labeled i.

Light delivery to the target employs components and techniques similar to those used in PDV [11]. The laser source for SMV is a single-frequency diode laser with Erbium doped fiber amplifier operating at $1550 \mathrm{~nm}$ wavelength ("laser" in Fig. 1). An advantage of the $1550 \mathrm{~nm}$ wavelength is the commercial availability of components such as highly directional and unpolarized optical circulators (labeled C in Fig. 1). Laser light entering port 1 of the circulator is transmitted to the target via port 2. The probe at the target is responsible for transmitting light to and collecting the return light from the target surface. Transmission to and from the target on one fiber enables a confocal imaging arrangement with high optical-collection efficiency.

One key difference when comparing SMV to PDV is a requirement in SMV that there is no back reflection from the probe itself. Since homodyne detection is simplest when detecting a single frequency, and since the laser light is delivered and returned on the same fiber, it is important that all SMV components between the circulator and the target have low back reflection. The power returned by the target must be at least $30 \mathrm{~dB}$ greater than the other reflections in the system to avoid generating additional beat frequencies that would make the data analysis unnecessarily more complicated. In our experiments, we obtained return values 
from the target surface in the range of -9 to $-16 \mathrm{~dB}$. We used components between the circulator and target surface (connectors and probe) that had back reflections $<-50 \mathrm{~dB}$.

The circulator routes the light from port 2 to port 3 with high directionality to the first 50/50 optical splitter. Splitter i divides the optical power between two distinct interferometers (labeled $i$ for interferometers $i=0$ or 1 in Fig. 1). The interferometers are unlike traditional VISARs in many respects. SMV is composed of two distinct Mach-Zehnder interferometers. The first splitter of each interferometer (labeled $p$ for the paths $p=0$ or 1 in Fig. 1) couples the input field into two fibers that propagate the field over two different interferometer paths. The fields are recombined at the couplers labeled $d$ (for detectors $d=0$ or 1 of each interferometer), and all transmitted power is detected using four high-speed photodetectors. The four optical output power levels are detected (producing four signals $\mathrm{D}_{\mathrm{d}}^{\mathrm{i}}, \mathrm{i}=0,1$ and $\mathrm{d}=0,1$ ) and the electronic signals are recorded.

In a manner reminiscent of traditional push pull VISARs [16], light-power fluctuations can be removed by detecting all of the light returned from the target in four detectors $\left(\mathrm{D}_{\mathrm{d}}^{\mathrm{i}}\right)$. Appendix B outlines in detail how the system design, system characterization, and data analysis remove unwanted amplitude variations due to target-reflection variation or extraneous light. Amplitude modulation can also arise from polarization modulation in a system with polarized components. To remove polarization dependence, we avoid the use of polarization dependent components. Each leg of an interferometer includes a polarization adjuster (PA; this is a miniature, manually operated, all-fiber, rotatable fiber-squeezer polarization controller, PolaRITE ${ }^{\mathrm{TM}}$ model PLC-M02, manufactured by General Photonics). The polarization adjusters between the couplers $p$ and $d$ are adjusted to compensate the interferometer-fiber birefringence and splitter polarization dependent losses (See appendix A). Polarization analyzers or scramblers are not used. The birefringence is adjusted to maximize the interference contrast and to remove target-polarization dependence from the signal amplitudes.

Each interferometer also contains one all-fiber phase shifter (PS). The phase shifter is a commercially available, electronically controlled piezo-fiber stretcher that can continuously introduce several wavelengths of optical delay. Operating the phase shifter produces interference fringes at the detectors that are used to characterize the interference amplitudes. 
For these experiments, we used New Focus model 1592 InGaAs detectors with a bandwidth of DC to more than $3.5 \mathrm{GHz}$. We checked the DC linearity of the detectors - the output responses deviated from linear by much less than $5 \%$ up to $1 \mathrm{~mW}$ at $1550 \mathrm{~nm}$. Experiment recordings were performed with TDS694c digital recorders (3 GHz bandwidth, 10 Gsamples/s) with digital-sample intervals of 100 ps or 200 ps. The overall bandwidth of this SMV system is expected to be better than $2 \mathrm{GHz}$.

Since the target-return reflection values are variable from target to target, and since the power levels on detectors need to be similar for each shot, a power monitor was used between the target and the interferometers (PM in Fig. 1) to establish the same power level to the interferometers and detectors for each shot. Before system adjustments were performed, the power to the target was adjusted to set the power at the PM to a value between $3 \mathrm{dBm}$ and $4 \mathrm{dBm}$ $(\sim 2$ to $2.5 \mathrm{~mW})$. The variable attenuators (VA) before the detectors were then adjusted upward to balance the fringe amplitudes during pre-shot characterization. Since the signal levels can increase in an experiment after shock-wave breakout, the initial power levels at the detectors were set to $<<1 \mathrm{~mW}$, i.e., well within the linear region of the detectors. While operating the phase shifters, the fringe maximum amplitudes were set to $\sim 0.1$ Volts into $50 \mathrm{Ohms}$ corresponding to $\sim 0.1 \mathrm{~mW}$ into each detector (for a total of about $\sim 0.2 \mathrm{~mW}$ into all detectors). The factor of ten power loss from 2 to $0.2 \mathrm{~mW}$ between the PM and the detectors arises from a combination of component losses and a conservative setting of the VAs to leave head room for the detector signal adjustments. From measured values for the target return-reflection loss between $-9 \mathrm{~dB}$ and $-16 \mathrm{~dB}$, the range of powers incident on targets may be inferred. We estimate the power levels incident on targets to be between $16 \mathrm{~mW}$ and $100 \mathrm{~mW}$ for all of the experiments described in this paper.

To reduce external influences on the interferometers such as vibration and temperature drifts, the interferometers were assembled in a rack mount box with packing foam to enclose the parts. After polarization adjustment, we found that the polarization controllers did not require readjustment in less than two days (the birefringence compensation was not strongly affected by temperature). A stronger drift occurs in the PZT-driven, fiber-stretcher phase shifters, which needed manual readjustment within the final seconds before an experiment. Rates of changes in experiments were billions of times greater than interferometer drifts. 
Immediately before each high-speed velocity measurement, the phase shifters were set to fixed values with an approximately-known interference phase relationship between the two interferometers. In practice, it is difficult to achieve an exact relative interference phase with two independent phase shifters. We have developed a method to analyze the recorded data using a nonlinear-equation minimization process for any two independent VPFs and any interference phase relationship between the two interferometers (see appendix B). Through simulation of data under various interferometer conditions we find that in the presence of noise the most robust and widely applicable configuration of the SMV instrument is to use two VPFs that are approximately equal and a relative interference-phase shift that is close to 90 degrees (similar to the ideal configuration in the push pull VISAR [16].)

\section{CALIBRATION AND CHARACTERIZATION}

Before performing a velocimetry experiment with SMV, several characterization operations must be carried out that are needed for data analysis. These operations include both one-time measurements and measurements that must be conducted prior to every experiment. One-time measurements include the detector characterization mentioned previously and interferometer calibration.

Interferometer calibration consists of determining the velocity that corresponds to a single interference fringe shift at the output of an interferometer, or rather determining the velocity per fringe constant (VPF). For velocities much less than the speed of light, the expression relating the Doppler frequency shift and surface velocity is

$$
\frac{\Delta f}{f}=\frac{2 V}{c},
$$

where $\Delta f$ is the frequency shift, $f$ is the frequency of the incident laser light, $V$ is the reflecting-interface velocity, and $c$ is the speed of light in vacuum.

At this point, other correction factors are sometimes included in the VPF. Additional corrections to (1) are well known for various effects such as index of refraction variation resulting from pressure waves in transparent tamping windows [18, 21,22,26-28] and Doppler shift variations at different angles of incidence [21]. These corrections depend on target parameters, are independent of the type of detection system and can be applied as various 
circumstances arise in target designs. We will not include additional corrections factors in the VPF for the instrument.

To obtain the VPF from (1), the frequency shift per fringe is needed. Traditionally, expression (1) is combined with another expression that obtains the frequency shift per fringe from other calibrated physical quantities such as interferometer path lengths to form an expression for the VPF $[15,16,21,26]$. When using this approach, a fiber based single-pass interferometer with difference $\Delta L$ between the path lengths has a frequency shift corresponding to one interferometer fringe described by

$$
\frac{\Delta f}{c}=\frac{1}{(n \Delta L)},
$$

where $n$ is the index of refraction within the interferometer paths. Since the index of refraction has chromatic dispersion $n=n(\lambda)$, a calibrated correction involving $d n / d \lambda$ is needed also.

Examination of the factor $c /(n \Delta L)$ in expression (1) reminds us that the frequency per fringe is related to a propagation time delay between the two interferometer paths as

$$
\tau=\frac{1}{\Delta f}
$$

Note that this time delay, $\tau$, is the optical phase delay difference associated with optical frequency and is not the group delay corresponding to amplitude modulation propagation delay that would be measured using an amplitude modulator. These two delays are not the same, but are related through the dispersion in $\mathrm{n}$ and can be different by several tenths of one percent in silica fiber. For the interferometer delays used in this work (several nanoseconds), the difference in phase and group delays is indistinguishable.

The availability of a tunable laser and high-speed detectors allowed a more direct approach to calibration. Instead of using length or group delay measurements and applying additional dispersion corrections, we measured the frequency shift corresponding to one fringe directly. Expressions (1) and (3) can be combined to form an expression for the VPF,

$$
V P F=\frac{\lambda}{2} \frac{1}{\tau}=\frac{\lambda}{2} \Delta f \quad[\mathrm{~m} / \mathrm{s} / \text { fringe }]
$$


where $\lambda$ is the laser wavelength in vacuum, and $\Delta f$ is the frequency change associated with one interferometer fringe. A calibration based on Eqn 4 does not require additional measurements of $n(\lambda)$ or $\Delta L$, which avoids the need to apply dispersion corrections. The physical effects of chromatic dispersion in the single mode fiber within the interferometer are included in the calibration. As an order of magnitude example of the sizes of parameters, for $\lambda=1550 \mathrm{~nm}$ and $\Delta L=1 \mathrm{~m}$ in silica fiber, $\tau \sim 5 \mathrm{~ns}, \Delta f \sim 200 \mathrm{MHz}$, and the velocity corresponding to one fringe would be VPF $\sim 155 \mathrm{~m} / \mathrm{s}$. Other choices for $\Delta L, \tau$ and the VPF are possible. To change the VPF for the SMV, fiber jumpers are added to alter the path length difference $\Delta L$ in the interferometers.

A choice for the path length difference, VPF and consequently for $\tau$ depends upon the expected velocity range and the desired levels of resolution in time and velocity in an experiment. Because of a common approximation used in the numerical analysis of VISAR data, the resolvable time difference in the computed velocity is usually not smaller than $\tau$ (see appendix B, Eqn B5). If a higher time resolution is desired, then the interferometer is configured with a shorter $\tau$ with correspondingly larger VPF. Alternatively, finer changes in velocity may be observed instead by reducing the VPF at the expense of time resolution.

After a choice for $\Delta L$ is made, measuring the frequency shift $\Delta f$ corresponding to one fringe gives the velocity per fringe constant directly. This calibration is performed individually on each interferometer after a fiber jumper has been inserted and the birefringence has been adjusted (see appendix A). As shown in Fig. 2, the calibration is accomplished by sending tunable laser light to the SMV interferometers, measuring the output power on the SMV detectors, and shifting the frequency of the tunable laser in amounts corresponding to one full interference fringe. The frequency shift is measured using heterodyne detection by comparing the tunable laser frequency to a fixed reference frequency. Our calibration set-up incorporates two lasers operating within a few gigahertz of each other near a wavelength of $1549.9 \mathrm{~nm}$. Heterodyne detection is accomplished by combining the tunable laser light and fixed frequency laser light at a 50 / 50 reflector, sending the combined fields to a high speed detector, and measuring the beat frequency with a spectrum analyzer. In Fig. 2, optical circulators are used as optical isolators to prevent back reflections to the lasers. Polarization adjusters (not shown) are used to maximize the heterodyne beat contrast. Repeated measurements were made over a span 
of ten fringes to verify linearity, i.e., that there are no higher order dispersion effects. The accuracy in the calibration depends on the spectrum analyzer calibration for frequency measurements in the $\mathrm{MHz}$ to $\mathrm{GHz}$ range and the value for the laser wavelength. For the data presented in this paper the interferometers were configured with two sets of VPFs of 122.8 and $122.6 \mathrm{~m} / \mathrm{s} /$ fringe $(\sim 158 \mathrm{MHz})$, or 241.6 and $239.6 \mathrm{~m} / \mathrm{s} /$ fringe (309 and $312 \mathrm{MHz}$ ).

Characterization measurements that must be conducted for each experiment include: timing characterization, zero-power detector offsets, the peak to peak signal values in the fringes while varying the phase shifter, and the power level returned from the target using the power monitor (PM in Fig. 1) during the peak to peak measurements and as the shot fires. Timing characterization is performed by inserting an electronic pulse generator (rise time $200 \mathrm{ps}$ ) and amplitude modulator ( $\mathrm{M}$ in Fig. 1) above the interferometers, and recording time differences between pulses at the recorders with various paths disconnected inside the interferometers (the use of fiber optic connectors is advantageous for timing). Timing characterization provides timing offset terms that are used to compensate for differences in the fiber and cable lengths from target to recorders. After balancing peak to peak interference amplitudes on the detectors using the phase shifters and variable attenuators, the peak to peak amplitudes are measured to obtain scale factors $\mathrm{B}_{\mathrm{d}}^{\mathrm{i}}$ needed to compensate for differences in interference amplitudes (see appendix B). Power levels at the power monitor PM are measured during characterization and at shot time. To summarize, the quantities that are needed during data analysis are: VPFs, propagation time differences, zero offsets, $\mathrm{B}_{\mathrm{d}}^{\mathrm{i}}$ scale factors, $\mathrm{PM}$ values, and an approximate estimate for the starting phases. The initial phases are refined using the shot data recorded just before breakout.

\section{EXPERIMENTAL RESULTS}

Before using the SMV on a large-scale shock physics experiment we used an exploding bridge wire (EBW) flier to demonstrate the operation of the single mode VISAR and to develop the characterization and analysis methods. An EBW uses a high voltage capacitive discharge in a spark gap to create a small ball of plasma. The EBW is mounted in a barrel with a diameter of several millimeters. The air space at the spark gap is confined by a metalized-plastic foil mounted in the barrel. To accelerate the foil flier we obtained a capacitive discharge unit (CDU) which would provide a one joule pulse to a graphite-pencil mark on a Risi RP-1 exploding bridge 
wire. Upon discharge in the confined-air space, the foil flier puffs up to velocities around 200 $\mathrm{m} / \mathrm{s}$.

The foil fliers were cut from a foil wrapper from a Strawberry Nutri-Grain bar to one quarter inch diameter disks. A foil flier consists of an aluminum layer sandwiched between layers of plastic with a total thickness of 40 microns. One side of the foil, the red side, was more reflective than the other, so we chose to focus the laser light onto this more reflective side of the foil using a fiber focuser or probe.

The probe used for the EBW experiments had a $-51 \mathrm{~dB}$ back reflection and a $16 \mathrm{~mm}$ focal length. The flier was arranged so that the least reflective side was 1 to $2 \mathrm{~mm}$ from the spark gap and the more reflective side of the foil was $16 \mathrm{~mm}$ from the focuser. To align the focuser to the foil flier, an optical back reflection meter was used to measure the return signal from the flier. In order to allow the probe to approach the optimum focus during the experiment the probe was pulled back from the position of maximum signal until the back reflection decreased $1.5 \mathrm{~dB}$. Typically, EBW shots were performed with a $-10 \mathrm{~dB}$ or better return reflection. For the EBW foil flyer experiments, the SMV velocimeter was configured with VPF constants close to 122 $\mathrm{m} / \mathrm{s} /$ fringe.

An example of raw EBW SMV data are shown in figure 3. Channels 1 and 2 were the outputs of the first interferometer and channels 3 and 4 were the second interferometer outputs. The outputs advance through more than 1.5 fringes before the surface reflection is lost (the sum of the signals abruptly falls after 2 microseconds on the time axis). In this data set the 180 degree phase shifts between black and red, and blue and green interferometer output traces is well illustrated near 1.5 microseconds. The approximately 90 degree phase shift between the two interferometers is shown between the pairs of interferometer outputs. The blue-green set of traces lags the red-black set of traces by approximately 90 degrees.

Compare the noise at the beginning and end of the traces. The noise is light related and anti-correlated across channels. This noise is reduced to much lower levels when the instrument is adjusted to remove interference. Hence, the noise that appears on each trace is dominated by high frequency phase noise in the laser with linewidth of $\sim 8 \mathrm{MHz}$. A narrower laser line width could be used to improve the velocity resolution. 
EBW flier experiments were configured, characterized, and fired several times. The duration between initial motion and when the light was extinguished was variable from about 0.1 to 1 microsecond. Velocity results for the two longest traces are shown in Fig. 4. The velocity traces have been offset vertically for clarity. All experiments began with a fast rise similar to the initial rise in Fig. 4 to $70 \mathrm{~m} / \mathrm{s}$. The topmost trace is the result obtained from an analysis of the data shown in figure 3. The larger amplitude variations in the lower trace result from one of the four detector signals going off scale from approximately 0.1 to 0.2 microseconds. After the initial fast rise, we see the signal flatten out for about half of a microsecond with total displacement less than the foil thickness $\left(<\left(0.5 \times 10^{-6} \mathrm{~s}\right)(50 \mathrm{~m} / \mathrm{s})=25\right.$ micrometers $)$. After the initial impulse in the foil, the velocity trace accelerates to $200 \mathrm{~m} / \mathrm{s}$ with a total displacement of several times the foil thickness before the foil disintegrates (perhaps the foil puffed out and popped). The foil motion is consistent with an initial shock front in the air impacting the foil followed by an additional acceleration due to pressurized (heated) air expanding behind the shock front.

Having developed repeatable experiment setup and characterization operations using foil fliers, we were ready to participate in a gas gun experiment. We wished to examine the accuracy of the method at higher velocities by a direct comparison between SMV and another velocimetry technique. To perform this comparison we inserted a single SMV probe into an existing gas gun experiment to measure the mass velocities at the back surface of a target after projectile impact (for details related to the projectile, target and shock-wave physics, see reference [29]).

As shown in Fig. 5, light returned from a single target probe was split and detected using an existing PDV detection channel [29] and the SMV velocimeter. We used a single $1550 \mathrm{~nm}$ laser to provide laser light for the probe (Fig. 5). A circulator was employed to couple laser light to and from a fiber probe mounted on the back of the target. The probe itself had the low back reflection required for SMV. Since the PDV requires un-shifted laser light for heterodyne detection, a fiber splitter/coupler was inserted immediately following the laser (before the circulator and target) to pick off some of the un-shifted laser light. A second coupler was used to combine un-shifted laser light and the Doppler shifted light from the target. This combination was sent to the PDV detection system and recorded using a $6 \mathrm{GHz}$ digital recording oscilloscope. For the SMV, only the Doppler shifted light was sent to the SMV and the 4 SMV signals were detected using a $3 \mathrm{GHz}$ recorder. 
The target included a LiF tamper window adjacent to the back of an aluminum target [29]. The changing optical delays at the target include contributions due to compression of the tamping window that produces an apparent interface velocity that is higher than actual $[18,21$, 22, 26-28]. An advantage of $\mathrm{LiF}$ is an index of refraction that varies linearly with density leading to a correction factor that is independent of pressure. For analysis of the gas gun experiment, a window-correction factor was used. In the gas gun experiment, the uncorrected VPF was $240 \mathrm{~m} / \mathrm{s}$. The corrected VPF with LiF window correction at $1550 \mathrm{~nm}$ [22] is 240/1.267 $=189.4 \mathrm{~m} / \mathrm{s}$. The correction factor accounts for changes needed in the Doppler shift expression (Eqn 1) and is independent of the instrumentation used to measure the wavelength. The same window correction factor was applied to both the PDV and SMV results.

The gas gun experiment results from both velocimeters are overlayed in the upper panel of Fig. 6. At shock-wave breakout, the aluminum-LiF interface jumped to $0.87 \mathrm{~km} / \mathrm{s}$ and increased to velocities over $1.2 \mathrm{~km} / \mathrm{sec}$. The SMV results were analyzed using the VPF calibration, correction factor, and the numerical methods described in appendix B. The PDV results were analyzed using a sliding-window frequency transform with a $6 \mathrm{~ns}$ transform window. The results in the upper panel overlay well. The lower panel of Fig. 6 shows the percent difference (residuals) between the two velocity traces. For the results at times between 5 ns and 0.37 microseconds after breakout, the residuals have an average deviation of $0.05 \%$, and standard deviation of 1.5 percent. Any non-linear stimulated Brillouin scattering would appear in the PDV results as an $11 \mathrm{GHz}$ side band on the PDV (above our bandwidth), but would not affect the frequency of the fundamental. Since the results from two fundamentally different techniques agree well, we believe that the combined phase errors due to any possible non-linear optical processes including Brillouin scattering were negligible in these experiments.

The results in Fig. 6 compare favorably up to 0.37 microseconds. After 0.37 microseconds the results diverged significantly. The experiment was arranged with the probe approximately $2 \mathrm{~mm}$ from the edge of the $\mathrm{LiF}$ window. Before 0.37 microseconds, the experiment is expected to be one-dimensional, and after 0.37 microseconds edge reflections produce a more complex three dimensional wave structure in the field of view of the probe. More work would be needed to understand why the two techniques disagree for the three dimensional wave structure in this experiment. However, within the one dimensional domain, 
the two techniques do agree to within $1.5 \%$ one standard deviation in the velocity values with a negligible mean difference (see the lower panel of Fig. 6).

For a VISAR that uses multimode fiber for light delivery with fiber dispersion comparable to the interferometer delay, an ambiguity or smearing of the number of fringes known as "lost fringes" can result during a rapid change in velocity. For the SMV using single mode fiber with $\mathrm{THz}$ of bandwidth, something different was observed in the interferometer outputs near breakout in Fig. 6.

Figure 7 shows the raw data for one of the SMV interferometers within several nanoseconds of the velocity jump in Fig. 6. In this experiment, the interferometer delay time was 3.2 ns (computed from Eqn 3 and the uncorrected VPF). During the interferometer delay time, the raw data exhibit $\sim 5$ oscillations with a 180 degree push-pull interference phase difference. The velocity jump of $0.87 \mathrm{~km} / \mathrm{s}$ at zero time in Fig. 6 is 4.6 times the VPF. In other words, during the first $3.5 \mathrm{~ns}$ after breakout, the fringes are not smeared by temporal dispersion and there is no ambiguity in the fringe count (for a more complete understanding of this comment see the text leading up to Eqn B5). Especially interesting, the shock-event signal rise

time of about $0.2 \mathrm{~ns}$ in the raw SMV data is not limited by the interferometer delay time of $3.2 \mathrm{~ns}$ The rise time of the first oscillation corresponds to the faster rise time of $\sim 0.2 \mathrm{~ns}$ one would expect in a PDV system with a $2 \mathrm{GHz}$ bandwidth.

\section{DISCUSSION}

We interpret the results in Figs. 6 and 7 as follows. After an input step function where the optical frequency shifts from $f_{0}$ to $f_{0}+f_{s}$, the interference phase at the output of the interferometer does not suddenly jump. The interference phase advances linearly as $\theta(t) \sim 2 \pi f_{s} t$ during the delay time $\tau^{i}$ while optical propagation in one path catches up with the other (see appendix B Eqn B3). During this catch up time, the interference terms in Eqns A2 or B2 oscillate at the shift frequency $f_{s}$. After the initial frequency jump and during the delay time while the two arms of the interferometer have two different frequencies, the two frequencies beat with a frequency shift of $1.4 \mathrm{GHz}$ corresponding to the jump velocity of approximately 0.87 $\mathrm{km} / \mathrm{s}$ - we interpret the SMV signals within the first interferometer delay time to be PDV-like 
heterodyne beat signal that occurs inside the interferometer until the frequency jump and reference frequencies have had time to travel over all paths to the last splitters (d).

Since PDV-like heterodyne beats are resolved in the interferometer within time $\tau^{i}$, there are no lost fringes and no fringe-count ambiguity for the $\sim 5$-fringe jump in Figures 6 and 7 . In this particular case, the additional information needed to determine the jump size was resolved in the high speed record of the interferometer data close to break out as shown in Fig. 7.

Resolving the fringes on a 0.2 ns time scale is possible in these data because we have avoided the $\sim 1$ ns modal distortion that would have washed out these fringes when using a traditional VISAR incorporating multimode delivery fiber. Modal distortions can also be avoided with free space optics. However, replacing multimode fiber with single mode fiber instead of redesigning with free space optics has practical advantages for facilities with containment barriers already incorporating multimode fiber for optical delivery.

Working at a longer wavelength $(1550 \mathrm{~nm}$ instead of the more traditional $532 \mathrm{~nm}$ or $514.5 \mathrm{~nm}$ ), does results in a longer $\tau$ for the interferometer at the same VPF, but clearly this does not mean that event-time resolution is made to suffer (it is improved). Event-time resolution should not be confused with the velocity-time resolution that results from the usual approximation employed in equation B5 when extracting the velocity. The approximation used in B5 merely reflects our present inability to mathematically invert the exact expression in equation B3 (which can be avoided also by another choice of VPF with a shorter $\tau^{i}$ value).

We have demonstrated the feasibility of an SMV for high velocity measurements at least as high as $1 \mathrm{~km} / \mathrm{sec}$ with an accuracy of at least 1.5 percent compared directly to a PDV while using a VISAR-like configuration that replaces the multimode mode fiber with single mode fiber. Since the SMV is a homodyne technique, it should be possible to measure much higher velocities with the same detection and recording electronics. The event-time resolution and velocity resolution are both comparable to that expected in a heterodyne system such as a PDV system with the same detection electronics. Since SMV needs four detectors and four recorder inputs for each target spot, whereas PDV uses one each, an engineering study of costs should be used to decide between the two methods in a range of velocities where electronics are available to PDV (less than $15 \mathrm{~km} / \mathrm{sec}$ ). 


\section{ACKNOWLEDGEMENTS}

This work was performed under the auspices of the United States Department of Energy at Lawrence Livermore National Laboratory under Contracts W-7405-ENG-48 and DE-AC5207NA27344. Some of the work in this paper was performed in part to satisfy the requirements for advancement to an M.S.E.E. degree from Cal Poly and is described in more detail in a thesis available in reference [32]. We would like to acknowledge useful conversations, technical assistance, and equipment loans from Neil Holmes, David Erskine, Ricky Chau, Elida White, Sam Weaver, Neal Hinsey, Steve Caldwell, Gino Mercado, Bob Naftzinger, Joe Bartolick, and Bruce Whitcomb. The gas gun shot data in this paper was obtained at LLNL in late 2007 with the assistance from many of these folks.

\section{APPENDIX A:}

\section{REMOVING POLARIZATION DEPENDENCE}

This appendix describes how the apparatus is aligned to achieve immunity to changes in polarization and a theoretical analysis of what is achieved during cancellation of the polarization dependence. While adjusting the polarization input to the interferometers, we found that the output powers can vary by as much as 100 percent. This test is performed by varying the polarization adjuster PA above the coupler labeled $\mathrm{i}$ in Fig. 1. Unpolarized single-mode fibers that were developed for high speed communications have weak birefringence with polarization mode dispersion values smaller than $\sim 1 \mathrm{ps} /(\mathrm{km})^{1 / 2}$. Our interferometers have legs with total length on the order of 1 to 2 meters. Although the birefringence is small, in one meter of fiber two polarization states can differ in propagation phase delay by approximately a few wavelengths (tens of femtoseconds). Since target-birefringence and polarization changes are known to occur in shock physics experiments [20], birefringence compensation is needed. Our approach was to compensate the interferometer birefringence in order to make SMV insensitive to polarization changes. We use polarization adjusters PA between the interferometer splitters (between splitters labeled $\mathrm{p}$ and $\mathrm{d}$ in Fig. 1) to remove polarization dependence at the detectors.

The power at detector $\mathrm{d}$ has the form 


$$
P_{d} \sim\left(\vec{E}_{p=0, d}+\vec{E}_{p=1, d}\right)^{*} \bullet\left(\vec{E}_{p=0, d}+\vec{E}_{p=1, d}\right),
$$

where $\vec{E}_{p, d}$ is the field propagated to detector $\mathrm{d}$ along path $\mathrm{p}$. For an ideal interferometer with no birefringence, (A1) reduces to a simple form for the detected signals D that are proportional to the output power levels P:

$$
\begin{aligned}
& D_{0}=G_{0} P_{0}=A_{0}+B_{0} \cos (\theta), \\
& D_{1}=G_{1} P_{1}=A_{1}-B_{1} \cos (\theta)
\end{aligned}
$$

where $\mathrm{G}$ is the detector gain. The interferometer outputs vary simply as $\cos (\theta)$, where $\theta$ is the phase delay difference between the two interferometer paths. For a slowly changing Doppler shift $\Delta f$, the interference phase advances in proportion to the frequency shift $\Delta \theta \sim \Delta f$. Numerical extraction of $\theta$ is required. With no compensation for birefringence in the interferometer, the form for the signals is much more complicated than A2; many terms are present that vary with input polarization. Polarization compensation is desired.

In practice, the operation we employ to remove the interferometer output dependence on input polarization is as follows. We display the two power levels $P_{0}$ and $P_{1}$ for two detectors of one interferometer on an oscilloscope that is set up to display a point with $P_{1}$ versus $P_{0}$ (commonly referred to as x-y display mode - see Fig. 2). The method is reminiscent of the method of displaying a circular Lissajous for free-space push-pull VISAR interferometers, but is not the same since we do not have the cosine and sine like signals. Using the phase shifter (PS) in Fig. 1, we vary the total phase difference between the two legs through several times $2 \pi$ (several wavelengths), and oscillate the interference terms $B \cos (\theta)$ at roughly $10 \mathrm{~Hz}$. While using a constant input power $P_{0}+P_{1}$, as $P_{0}$ increases, $P_{1}$ decreases. I.e., the display exhibits a downward sloping oscillating trace. While oscillating the $B \cos (\theta)$ terms with the phase shifter, we slowly vary the input polarization using the polarization adjuster above splitter i (in Fig. 1). When the birefringence has not been properly adjusted, the slope and end points of the display will vary with the input polarization. While oscillating the phase shifter and slowly varying the input polarization, we then adjust the polarization adjusters PA in each leg of an interferometer (between $\mathrm{p}$ and $\mathrm{d}$ in Fig. 1). We have developed procedures that lead to a straight line display with a minimum of variation of the slope and end points while the input polarization varies. In other words, by proper adjustment of two polarization adjusters, i.e., one per leg of an 
interferometer, we are able to reduce the output dependence on input polarization to low levels with variation typically less than 1.5 percent. The fiber splitter/couplers used in this work had a polarization dependent loss specification of 3 percent. We wished to understand how this compensation occurs.

In the remainder of this appendix, we examine how the birefringence transforms the input field, and what adjustments are required to remove the polarization dependence. The fields in Eqn A1 are related to an input field that has been transformed by the splitters, fibers, and onefiber devices. The fiber paths can be considered to consist of a series of short legs of length $\mathrm{L}_{\mathrm{i}}$ with outputs connected to subsequent inputs. For this analysis, we consider the fiber segments to be weakly birefringent and to have no power losses and no scattering or power coupling between the polarization eigenmodes. At each connection, local (different) coordinate axes may be found such that the birefringence transforms the input field $\vec{E}=\left(E_{x}, E_{y}\right)^{T}$ to an output field $\left(E_{x^{\prime}}, E_{y^{\prime}}\right)^{T}$ by a transformation of the form $e^{i \Delta_{i}} B_{i}$ where

$$
B_{i}=\left(\begin{array}{cc}
e^{+i \delta_{i}} & 0 \\
0 & e^{-i \delta_{i}}
\end{array}\right)
$$

i.e., input and output local coordinates can be selected that make $e^{i \Delta_{i}} B_{i}$ diagonal. I.e., for a short leg, polarization eigenmodes can be found that are not mixed but are phase delayed by $\left(\Delta_{i} \pm \delta_{i}\right)$. A3 is written so that the total phase delays for input components $E_{x}$ and $E_{y}$ split the phase difference $2 \delta$ symmetrically about $\Delta$ :

$$
\begin{aligned}
& \left(\Delta_{i}+\delta_{i}\right)=n_{x}\left(2 \pi L_{i} / \lambda\right) \\
& \left(\Delta_{i}-\delta_{i}\right)=n_{y}\left(2 \pi L_{i} / \lambda\right)
\end{aligned}
$$

The phase delays results from the number of wavelengths dividing the segment length. The vacuum wavelength $\lambda$ is divided by the different effective indices of refraction $n_{x}$ and $n_{y}$ in $\mathrm{x}$ or y polarization. Since each leg may be given an arbitrary rotation or twist relative to the others, each set of input-field components may be expressed in terms of the previous-output's field components transformed by a coordinate rotation through angle $\alpha_{i}$ by rotation transformation $R_{i}=R\left(\alpha_{i}\right)$. The complete transformation of a field through a short, single-fiber segment consists of a rotation and differential phase lag: $e^{i \Delta_{i}}\left(B_{i} R_{i}\right)$. 
For a two-fiber power splitter/coupler, the assumption of no-power coupling between modes is relaxed. The cores of the fibers are in close proximity. A fraction of the power from one fiber couples to the other via an overlap of the field from the first fiber with the core of the second fiber over a short distance $(\sim 1 \mathrm{~cm})$. Because there may be small amounts of birefringence in the coupling region, the transformations of input to output fields (in local coordinates) have slightly different phase delays $\varphi_{x}$ and $\varphi_{y}$. The output fields have the for form [30]:

$$
\begin{aligned}
& \left(\begin{array}{l}
E_{x^{\prime}} \\
E_{y^{\prime}}
\end{array}\right)_{\text {same }}=\left[\operatorname{Re}\left(\begin{array}{cc}
e^{i \varphi_{x}} & 0 \\
0 & e^{i \varphi_{y}}
\end{array}\right)\right\rfloor\left(\begin{array}{c}
E_{x} \\
E_{y}
\end{array}\right) \\
& \left(\begin{array}{c}
E_{x^{\prime}} \\
E_{y^{\prime}}
\end{array}\right)_{\text {opposite }}=\left[i \operatorname{Im}\left(\begin{array}{cc}
e^{i \varphi_{x}} & 0 \\
0 & e^{i \varphi_{y}}
\end{array}\right)\right]\left(\begin{array}{c}
E_{x} \\
E_{y}
\end{array}\right) .
\end{aligned}
$$

$\vec{E}=\left(E_{x}, E_{y}\right)^{T}$ is an input field. $\left(E_{x^{\prime}} E_{y^{\prime}}\right)^{T}$ same and $\left(E_{x^{\prime}} E_{y^{\prime}}\right)^{T}$ opposite are the output fields on the same fiber as the input fiber, or the opposite fiber. The diagonal components decrease as $\cos \left(\varphi_{i}\right)$ or increase as $\sin \left(\varphi_{i}\right)$ with the different propagation delays $\varphi_{x}$ and $\varphi_{y}$ on the same or opposite fibers, respectively. The birefringence due to slightly different phase delays $\varphi_{x}$ and $\varphi_{y}$ in a coupler defines a pair of polarization eigenmodes with orientations defined relative to two different fibers. A 50 percent power splitter has $\varphi_{i} \bmod 2 \pi \approx \pi / 4$. When the phases modulo $2 \pi$ are not precisely equal to $\pi / 4$, Eqn A5 yields a small residual polarization dependence in the output power of the form

$$
P_{\text {out }}=\frac{1}{2} P_{i n}\left[1 \pm \frac{\left(\varepsilon_{x} E_{x}^{*} E_{x}+\varepsilon_{y} E_{y}^{*} E_{y}\right)}{\left(E_{x}^{*} E_{x}+E_{y}^{*} E_{y}\right)}\right],
$$

where $\varepsilon_{x}$ and $\varepsilon_{y}$ are small factors dependent on the phase differences $\varphi_{x}$ and $\varphi_{y}$. In other words, birefringence in the splitter will produce a small polarization dependent loss (PDL) that oscillates as 


$$
\sim \frac{1}{2}\left(\varepsilon_{x}-\varepsilon_{y}\right) \sin ^{2}\left(\phi_{p o l}\right)
$$

with changes in the linear-polarization input angle $\phi_{p o l}$ with peak to peak magnitude $\varepsilon=\left|\varepsilon_{x}-\varepsilon_{y}\right|$.

For the work in this paper, we used 50 percent couplers with a $P D L=\varepsilon=\left|\varepsilon_{x}-\varepsilon_{y}\right|<0.15 \mathrm{~dB}=3$ percent. Typically, we have been able to reduce the polarization dependence in the interferometer outputs to less than 1.5 percent with splitters having $\varepsilon=3$ percent.

The power at each detector can be computed from a series of transforms involving the diagonal form of the differential phase delay Eqn A3, the total phase delay and segment rotation $e^{i \Delta_{i}}\left(B_{i} R_{i}\right)$, and polarization dependent splitter expressions Eqn A5. Each field that is input to the first splitter of an interferometer (at $p$ in Fig. 1) travels along two paths $p=0$ and $p=1$ and arrives at a detector as a sum of two expressions each of the form

$$
\begin{gathered}
e^{i \Delta}[a]\left[\left(R_{n}\right)\left(B_{n-1} R_{n-1}\right) \cdots\left(M_{A}\right) \cdots\left(B_{2} R_{2}\right)\left(B_{1} R_{1}\right)\right][b] \vec{E} \\
=e^{i \Delta}[a]\left[M_{\text {Total }}\right][b] \vec{E}
\end{gathered}
$$

Here, [a] and [b] are the splitter transforms in A5 selected depending on whether the output is on the opposite fiber or the same fiber as the input fiber. The total phase delay is $\Delta=\Delta_{n}+\cdots+\Delta_{1}$ for the path. The operator $\left(M_{A}\right)$ is the transform matrix for the polarization adjuster (PA) in the selected path. Not shown in A7 are four pairs of labels (same, opposite) on the splitters [a] and [b], resulting in four expressions of the form (A7) involving two polarization adjusters $\left(M_{A}\right)$ for the two paths. Since each of the BR are unitary, the total fiber transformations between the two splitter-transforms [a] and [b] combine to form two different unitary matrices denoted [ $\left.M_{\text {Total }}\right]$.

Using the algebraic expressions described above, we explored theoretically what needs to occur when the polarization adjusters PA are manipulated to eliminate polarization dependence in the power levels $P_{0}$ and $P_{1}$ at the detectors. Using a pair of fields of the form A7, the total power at detector $\mathrm{d}$ can be computed from Eqn A1. When the product in A1 is expanded, in general there are polarization cross terms of the form $E_{x}^{*} E_{y}$ that mix $\mathrm{x}$ and $\mathrm{y}$ input polarization 
components at the detectors. These cross terms oscillate twice as fast the polarization angle; they are difficult to eliminate by any means other than to rotate polarization adjusters $M_{A}$ in both legs in such a way as to un-mix the $\mathrm{x}$ and $\mathrm{y}$ field components from both paths going into a second splitter - a polarization adjuster in each leg is useful. The magnitude of the polarization cross terms $E_{x}^{*} E_{y}$ may be reduced to small error terms when the polarization transforms $M_{A}$ in each interferometer arm are adjusted so that the total transforms between the brackets $\left[M_{\text {Total }}\right]$ in A7 closely aligns the splitter eigenmode polarizations of the $\mathrm{p}$ and $\mathrm{d}$ splitters for both paths at the same time, i.e., when the two total transforms $\left[M_{\text {Total }}\right]$ are both diagonal. In other words, the paths between the splitters are aligned to the preferred local axes at the splitters. When diagonal, they are both similar in form to the diagonal birefringence in A3, i.e., $M_{\text {Total }}=B_{\text {Total }}$. We arrive at a requirement for the instrument: a polarization adjuster is required in each interferometer path.

Beyond diagonalizing $M_{\text {Total }}=B_{\text {Total }}$ in both legs to remove polarization cross terms, one additional adjustment is needed to simplify the powers $P_{0}$ and $P_{1}$ to the simple form shown in (A2). First, without further adjustment of the phase difference in $B_{\text {Total }}$, taking the product A1 with A7 and $M_{\text {Total }}=B_{\text {Total }}$ can produce several polarization dependent terms in A2 that depend on the interference phase as $\cos \left(\theta+\theta_{0}\right)$ with different relative phases $\theta_{0}$. The phases $\theta_{0}$ can be aligned (making the terms equivalent to one cosine term) when the overall $B_{T}$ is set equal to $\pm I$ (I is the identity transform). $\quad M_{\text {Total }}=B_{\text {Total }}= \pm I$ may be achieved by a series of possible fullwave multiples between the $\mathrm{x}$ and $\mathrm{y}$ polarizations, i.e.,

$$
2 \delta_{T}=2 \pi m, m=0, \pm 1, \pm 2, \ldots
$$

When the $M_{\text {Total }}=B_{\text {Total }}$ are set close to $I$, but not precisely $I$, small residual polarization dependent terms in $P_{0}$ and $P_{1}$ remain that are first order in the PDL's, $\sim O(\varepsilon)$. Under the assumptions in this appendix (no loss and no coupling except at couplers), a remarkable feature of the design shown in Fig. 1 appears when $\left[M_{\text {Total }}\right]$ is set precisely equal to $\pm I$, namely, all of the first order error terms of order $O(\varepsilon)$ in $P_{0}$ and $P_{1}$ cancel and the lowest order of the remaining error terms is of second order $O\left(\varepsilon^{2}\right)$ in the PDLs of the splitters - the total interferometer polarization dependence can be made smaller than the polarization dependent 
losses of each of the splitters. This cancellation occurs because each of the power redirection terms of the two splitters has a compensating term that cancels when the splitters are nearly $50 \%$ splitters. Using splitters with PDLs of $\varepsilon=3$ percent, we expect that it should be possible to adjust the polarization dependent errors in $P_{0}$ and $P_{1}$ to be less than $\sim \varepsilon^{2}=0.1$ percent due to the cancellation of first order terms.

In order to achieve this level of rejection of polarization effects, it would be sufficient to maintain unmixed polarization eigenmodes throughout each leg as is often an implicit feature of free space interferometers, but strangely, maintaining eigenmode separation at intermediate locations is not necessary. Each of the transforms $\left(B_{i} R_{i}\right)$ have off diagonal components and may mix the polarization components at the input to each short segment. It is not necessary that this mixing be avoided at arbitrary locations since we can always set the total transforms at the final couplers to $M_{\text {Total }}= \pm I$; we only require that we can set the polarization adjuster so that when all the fields do arrive at the inputs of the last couplers, then the output polarization components emerge with no cross terms. Note that setting

$$
\begin{aligned}
& M_{T}= \pm I \\
& \text { implies } \delta_{T}=\pi m, m=0, \pm 1, \pm 2, \ldots
\end{aligned}
$$

Any of these $\delta_{T}$ serve our purpose to simplify the powers to the form in Eqn A2 with a single term equivalent to $B \cos (\theta)$. In other words, operationally achieving $M_{T}= \pm I$ does not guarantee that $\mathrm{m}$ and the total birefringence are zero. Since we avoid tight turns in the construction of the interferometers, we expect $m$ to be a small number and the birefringence phase difference to be an integral number of optical cycles, but we cannot tell if the m values are uniquely zero after adjustment.

The polarization alignment adjustments are performed at one wavelength. Fortunately also for a wide range of frequencies the ambiguity in the value of $\mathrm{m}$ (for small $\mathrm{m}$ ) is also unimportant when $B \cos (\theta)$ advances as the frequency shifts. For velocities on the order of $\sim 10$ $\mathrm{km} / \mathrm{sec}$, the frequency will shift on the order of $\sim 20 \mathrm{GHz}$ out of $\sim 200 \mathrm{THz}$. Since the relative change in the wavelength is small, $\Delta \lambda / \lambda_{0} \leq 10^{-4}$, the interference phase differences $\pi m \lambda_{0} / \lambda$ are affected negligibly as the wavelength changes, and the different $\cos \left(\theta+\theta_{0}\right)$ interference terms do not dephase measurably. A requirement to remove polarization effects is to be able to set the 
polarization adjusters in each leg so that the unavoidable field polarization mixing along each leg emerges from the second splitter unmixed again, but eigenmode separation at all points is not necessary. The design that we describe in this paper has an advantage of being capable of removing the polarization dependence, and canceling to first order all polarization dependent losses of the splitters.

\section{APPENDIX B:}

\section{SYSTEM CHARACTERIZATION AND DATA ANALYSIS}

In this appendix, we focus on understanding what characterization information is needed in order to convert recorded data to mass velocities. The analysis in this section has similarities to the analysis in traditional push-pull Michelson interferometer based VISARs [16, 21], but requires a generalization needed for the SMV as described below. This generalization occurs at a point where constraints on VPFs and phases need to be relaxed. In this section, we assume that the interferometer birefringence and polarization controllers have been adjusted operationally to remove dependence on input polarization as described in the third paragraph in appendix A. In the remainder of this work, we ignore small errors ( 0.1 percent) due to second order polarization dependence. We begin this section by computing the fields and powers, including propagation delays and the dependence of the interference phase on the optical frequency.

As a starting point for computing the fields, we will introduce are large number of polarization-independent transmission factors. Our goal is to remove the need for careful characterization of a large number of transmission factors. A goal for this development is to examine what system-characterization measurements can be made to cause these parameters to drop out of the result.

See Fig. 1 for the superscript and subscript labeling convention involving labels i, p, and $\mathrm{d}$ (which label interferometers, paths, and detectors). The first splitter has indices $\mathrm{i}=0,1$ which are also the interferometer labels. The interferometer label $\mathrm{i}$ is used below as a superscript. The fiber paths in each interferometer have labels $p=0,1$. The detectors have the same labeling $d=$ 0,1 as the labeling on the last coupler.

In the last section (Appendix A) we assumed no real power losses for the sake of analyzing how the polarization dependence can be removed. In this section we assume the 
polarization dependence was adjusted to be negligible (second paragraph of Appendix A). We treat each splitter/coupler output as having a polarization-independent transmittance factor that may contain loss (i.e., in this appendix the transmittances are scalar values rather than starting with matrix transforms A5 or A7). For the following, the power transmittances for the splitter and subsequent fiber legs are labeled with i, p, and d; will have a numerical values close to (1/2); and will be denoted as $\left(c^{i}\right)^{2},\left(b_{p}^{i}\right)^{2}$, and $\left(a_{d}^{i}\right)^{2} \cdot\left(c^{i}\right)^{2} \cong 1 / 2$ are the power transmittance values into each of the interferometers labeled i. Within the ith interferometer, $\left(b_{p}^{i}\right)^{2} \cong 1 / 2$ and $\left(a_{d}^{i}\right)^{2} \cong 1 / 2$ are the transmittances directing power along paths $\mathrm{p}$ and to detectors $\mathrm{d}$, respectively.

The field leaving the target $E(t)$ has a time varying amplitude and phase. The fields arriving at the detectors $D_{d}^{i}=D_{0}^{0}, D_{1}^{0}, D_{0}^{1}$, or $D_{1}^{1}$ at laboratory time $\mathrm{t}$ are superpositions of fields resembling the $\mathrm{E}$ that left target at different earlier times, $E\left(t-t_{p d}^{i}\right)$, were $t_{p d}^{i}$ are the total propagation times from the target surface to the recorders along different paths $i \rightarrow p \rightarrow d$ through the interferometers. (In what follows, we will use $t_{p d}^{i}$ interchangeably for phase delays and group delays, will point out when we mean one or the other, and finish by arguing that for this work the distinction is unimportant). There are $2 \times 2 \times 2=8$ propagation times $t_{p d}^{i}$ and 8 lagging copies of E. The superposed fields that deliver power to the detectors and produce signals $D_{0}^{i}$ or $D_{1}^{i}$ for interferometer i depend on the splitter transmittances and delay times as

$$
\begin{aligned}
& E_{d=0}^{i}(t)=c^{i} \quad b_{0}^{i} \quad a_{0}^{i} E\left(t-t_{00}^{i}\right)+c^{i} b_{1}^{i} a_{0}^{i} E\left(t-t_{10}^{i}\right) \\
& E_{d=1}^{i}(t)=c^{i} \quad b_{0}^{i} \quad a_{1}^{i} E\left(t-t_{01}^{i}\right)+c^{i} b_{1}^{i} a_{1}^{i} E\left(t-t_{11}^{i}\right)
\end{aligned}
$$

The optical power at detector $\mathrm{d}$ of interferometer $\mathrm{i}$ is proportional to $P_{d}^{i} \sim E_{d}^{i *} E_{d}^{i}$. The powers are detected with linear gains $G_{d}^{i}$ that produce electronic signals $D_{d}^{i}=G_{d}^{i} P_{d}^{i}$. Hence, the signals are 


$$
\begin{aligned}
& D_{d=0}^{i}=G_{d=0}^{i}\left\{\left(c^{i} b_{0}^{i} a_{0}^{i}\right)^{2} P_{\text {inc }+c}\left(t-t_{00}^{i}\right)+\left(c^{i} b_{1}^{i} a_{0}^{i}\right)^{2} P_{\text {inc }+c}\left(t-t_{10}^{i}\right)+2\left(c^{i}\right)^{2} b_{0}^{i} b_{1}^{i}\left(a_{0}^{i}\right)^{2}\left[P_{c}\left(t-t_{00}^{i}\right) P_{c}\left(t-t_{10}^{i}\right)\right]^{1 / 2} \cos \theta(t)_{d=0}^{i}\right\} \\
& D_{d=1}^{i}=G_{d=1}^{i}\left\{\left(c^{i} b_{0}^{i} a_{1}^{i}\right)^{2} P_{\text {inc c c }}\left(t-t_{01}^{i}\right)+\left(c^{i} b_{1}^{i} a_{1}^{i}\right)^{2} P_{\text {inc }+c}\left(t-t_{11}^{i}\right)-2\left(c^{i}\right)^{2} b_{0}^{i} b_{1}^{i}\left(a_{1}^{i}\right)^{2}\left[P_{c}\left(t-t_{01}^{i}\right) P_{c}\left(t-t_{11}^{i}\right)\right]^{1 / 2} \cos \theta(t)_{d=1}^{i}\right\}
\end{aligned}
$$

The powers $P_{i n c+c}$ and $P_{c}$ are total power levels returned from the target through the circulator to splitter i. The subscripts refer to two possible types of light in the system, namely, incoherent light (inc) from extraneous sources and the coherent light ( c ) from the laser. The two leading terms are proportional to the sum $P_{i n c+c}$ of the coherent laser source, and any additional incoherent (non interfering) sources. The interference terms are only proportional to the coherent laser power $P_{c}$ and do not include a contribution by sources with a coherence length much shorter than then interferometer delay (this convenient short hand notation is borrowed from Ref [21]). The power factors on the interference terms $\cos \theta_{d}{ }^{i}(t)$ contain only the coherent (interfering) part of the total power $P_{c}$. Note that the delay times $t_{p d}^{i}$ included in power factors $P\left(t-t_{p d}^{i}\right)$ are the group delays related to the propagation velocity of amplitude modulation in the optical field.

The different phase-propagation delays result in the interference factors $\cos \theta_{d}^{i}(t)$. Without approximation, the phase difference $\theta_{d}^{i}(t)$ is an integral of the optical frequency accumulated during a short time interval corresponding to the small difference in propagation times through the interferometer $[15,18,31]$ :

$$
\begin{aligned}
& \theta_{d}^{i}(t)=\int_{\mathrm{t}_{\mathrm{a}}}^{\mathrm{t}} d u \omega(u), \\
& \text { where } \mathrm{t}_{\mathrm{a}}^{\mathrm{a}}=\mathrm{t}-\mathrm{t}_{1, \mathrm{~d}}^{\mathrm{i}}, \mathrm{t}_{\mathrm{b}}=\mathrm{t}-\mathrm{t}_{0, \mathrm{~d}}^{\mathrm{i}}
\end{aligned}
$$

$\omega$ is $2 \pi$ times the optical frequency, and $\mathrm{u}$ is a dummy variable for time. In (B3), the times $t_{p d}^{i}$ have a subtly different meaning than the times $P\left(t-t_{p d}^{i}\right)$ in the power expressions - they are phase propagation time delays from target to detectors. (As will be seen after some algebraic 
manipulation, the group delays will divide out, while the total phase delay associated with the interference factors will remain). The interface phase at time t has contributions from frequencies reflected from the target at earlier times $t_{a}$ and $t_{b}$. When all other paths are equal, the integration interval $t_{b}-t_{a}$ has a small value

$$
\tau_{d}^{i}=\mathrm{t}_{\mathrm{p}=1, \mathrm{~d}}^{\mathrm{i}}-\mathrm{t}_{\mathrm{p}=0, \mathrm{~d}}^{\mathrm{i}}
$$

which is the interferometer delay difference between two paths from target to recorders.

From the usual (non-relativistic) Doppler approximation, the frequency shift is related to the velocity by $\omega_{S}=2 \omega_{0} V / c$, where $\omega_{0}$ is $2 \pi$ times the un-shifted laser frequency. The goal for data analysis is to extract the interference phase $\theta_{d}^{i}(t)$ from the four detected signals $D_{0,}^{0} D_{1}^{0} D_{0}^{1}$, and $D_{1}^{1}$ in equations (B2), extract the frequency shift $\omega_{S}$ from (B3), and compute the particle velocity from $\omega_{S}$.

Inverting the integral transform in B3 to obtain $\omega_{S}$ directly is problematic. VISAR data are often analyzed using an approximation that is sometimes referred to as the "VISAR approximation" [21], where the integral function in (B3) is replaced heuristically by an average value for the frequency shift averaged over the interferometer time delay:

$$
\theta_{d}^{i}(t)=\bar{\omega}_{s}(t-\bar{t}) \tau_{d}^{i}+\theta_{d}^{i}(0)
$$

where $\bar{\omega}_{S}$ is the frequency shift averaged over an interval $\tau^{i}$ and $\bar{t}=\left(\mathrm{t}_{\mathrm{p}=0, \mathrm{~d}}^{\mathrm{i}}+\mathrm{t}_{\mathrm{p}=1, \mathrm{~d}}^{\mathrm{i}}\right) / 2$ is the average phase-propagation time from target to recorders. Before shock breakout at times $\mathrm{t}$ $<<0$ where the surface has not yet moved, there is an arbitrary constant interference phase $\theta_{d}^{i}(0)$ in B5 equivalent to $\theta_{d}^{i}(0)=\omega_{0} \tau^{i} \bmod 2 \pi$. This starting phase depends sensitively on the value of the interferometer delay $\left(\omega_{0} \tau^{i}\right.$ is a large number from which only a small remainder $\theta_{d}^{i}(0)=\omega_{0} \tau^{i} \bmod 2 \pi$ is important). After the surface moves, the signals arrive at the recorders 
after the average propagation time $\bar{t}$, and the additional phase shift $\bar{\omega}_{S}(t-\bar{t}) \tau^{i}$ is the frequency shift times the interferometer delay $\tau^{i}$ (averaged during interval $\tau^{i}$ ). People using this approximation need to realize that it ignores the reality that the interference phase does not change abruptly, and that for any abrupt frequency shift input to the integral in B3 (of any size), the output phase takes the same finite time $\tau^{i}$ to advance to a new value.

It is possible to consider a comparison of the total phase-propagation time for two completely different measurement channels from a complete characterization of propagation times $\bar{t}$ using pulse (amplitude modulated) group delay times. For the comparison of PDV and SMV data in this work (Fig. 6) we instead aligned the time axes by noting the location of the abrupt shock wave breakouts in the two separate data sets. For future work involving more precise timing comparisons of homodyne and heterodyne channels, additional considerations may be needed to address the difference in phase and group propagation delays for the two systems (we will ignore this distinction for now).

To make further progress towards extracting $\theta_{d}^{i}(t)$ from B2, we need additional system characterization information. Zero offsets are measured with the laser turned off. The zero offsets are subtracted from the signal amplitudes in B2. During system characterization, we vary $\theta_{d}{ }_{d}^{i}(t)$ at slow speeds over several multiplies of $2 \pi$ using a fiber phase shifter and observe the oscillating amplitudes on the $\cos \theta_{d}^{i}(t)$ terms. While path-length changes are made, recordings are obtained while holding $P_{i+c}=$ const, $P_{c}=$ const , with $\theta_{0}^{i}(t)=\theta_{1}^{i}(t)$. The slow speed signal recordings have a form shown in Eqn A2. From the slow speed recordings, we obtain values for the scale factors $B_{d}^{i}$ in A2. We can remove the dependence on many of the unknown transmission factors $\left(c^{i}\right)^{2},\left(b_{p}^{i}\right)^{2}$, and $\left(a_{d}^{i}\right)^{2}$ and gains $G_{d}^{i}$ in B2 by rescaling the high speed recordings by the measured $B_{d}^{i}$ factors. It is important in VISARs that the gains $G_{d}^{i}$ are linear and have similar frequency dependence; if the frequency dependence is similar, the gains drop out when dividing by $B_{d}^{i}$ :

$$
\frac{D_{0}^{0}}{B_{0}^{0}}=\frac{1}{P_{c}(0)} \frac{1}{2 b_{0} b_{1}}\left\{b_{0}^{2} P_{i n c+c}\left(t-t_{00}\right)+b_{1}^{2} P_{i n c+c}\left(t-t_{10}\right)\right\}+\frac{1}{P_{c}(0)}\left[P_{c}\left(t-t_{00}\right) P_{c}\left(t-t_{10}\right)\right]^{1 / 2} \cos \theta_{0}^{0}
$$




$$
\frac{D_{1}^{0}}{B_{1}^{0}}=\frac{1}{P_{c}(0)} \frac{1}{2 b_{0} b_{1}}\left\{b_{0}^{2} P_{\text {inc } c}\left(t-t_{01}\right)+b_{1}^{2} P_{i n c+c}\left(t-t_{11}\right)\right\}-\frac{1}{P_{c}(0)}\left[P_{c}\left(t-t_{01}\right) P_{c}\left(t-t_{11}\right)\right]^{1 / 2} \cos \theta_{1}^{0}
$$

Similar expressions are obtained for the second interferometer with superscript 1. (In the analysis, we offset the recorded signals D by their zero offsets before rescaling). Unknowns $\left(c^{i}\right)^{2},\left(a_{d}^{i}\right)^{2}$ and gains $G_{d}^{i}$ are replaced by a measured parameter $B_{d}^{i}$.

The leading terms in B7 are similar. We would like to subtract and divide expressions such as B7 in order to remove the time varying total powers $P\left(t-t_{p d}^{i}\right)$. At a resolution of $100 \mathrm{ps}$ corresponding to $\sim 2 \mathrm{~cm}$ of cable length, it is impractical to require the sums of all cable delays both inside and outside the interferometers to be precisely equal. Instead, the different delay times in the power factors $P\left(t-t_{p d}^{i}\right)$ in B7 can be aligned numerically by shifting the recorded signals by amounts obtained during system timing characterization. The time bases in the digitally recorded data for each $D_{d}^{i}$ are sampled at sample intervals of $\Delta t$. The recordings can be shifted by multiples of the sample interval, $D_{d}^{i}(t-n \Delta t)$ before subtracting and dividing expressions B7. To characterize the time differences, we inserted a pulsed Mach Zehnder modulator before the splitters ( $M$ in Fig. 1).

Since it is not immediately obvious what constraints may be present already on the eight different times $t_{p d}^{i}$, or whether it is even possible to align all of the power factors in B7, we examine these constraints now to see which timing information is needed. One constraint on the times can be found by examining A8, B3 and B4. For the $i$ th interferometer, we expect that the interferometer phase-delay differences associated with either of it's detectors, $\tau_{0}^{\mathrm{i}}=\mathrm{t}_{10}^{\mathrm{i}}-\mathrm{t}_{00}^{\mathrm{i}}$ or $\tau_{1}^{\mathrm{i}}=\mathrm{t}_{11}^{\mathrm{i}}-\mathrm{t}_{01}^{\mathrm{i}}$, to be the same to within a small integer multiple of the optical cycle time or $\sim 5$ fs (see comments after A8). With negligible error, we can set $\tau^{\mathrm{i}}=\tau_{0}^{\mathrm{i}}=\mathrm{t}_{10}^{\mathrm{i}}-\mathrm{t}_{00}^{\mathrm{i}}=\tau_{1}^{\mathrm{i}}=\mathrm{t}_{11}^{\mathrm{i}}-\mathrm{t}_{01}^{\mathrm{i}}$, drop the subscript $\mathrm{d}$ on the phase functions and set $\theta^{i}=\theta_{d}^{i}$ equal in B5 and B7.

To avoid interference effects in the timing measurement, during timing we disconnected the $p=1$ paths in the interferometers, and measured the pulse-propagation time delay differences 
for the $\mathrm{p}=0$ paths for all detectors on a single four channel digital recorder (with one time base). These measurements were performed with an accuracy of $30 \mathrm{ps.} \mathrm{We} \mathrm{then} \mathrm{shift} \mathrm{and} \mathrm{align} \mathrm{the}$ time bases of the recordings $\mathrm{D}$ by multiplies of the sample interval time, to align the $\mathrm{p}=0$ times, which sets $t_{00}^{0}=t_{01}^{0}=t_{00}^{1}=t_{01}^{1}$ between the two interferometers to within one recorder sample interval (200 ps or better). Since for one interferometer $i$ the loop time around the interferometer has a single value, i.e., $\tau^{\mathrm{i}}=\tau_{0}^{\mathrm{i}}=\mathrm{t}_{10}^{\mathrm{i}}-\mathrm{t}_{00}^{\mathrm{i}}=\tau_{1}^{\mathrm{i}}=\mathrm{t}_{11}^{\mathrm{i}}-\mathrm{t}_{01}^{\mathrm{i}}$, shifting the time bases to align the $\mathrm{p}=0$ paths also aligns the $\mathrm{p}=1$ paths for the two detectors in each interferometer, i.e., $t_{10}^{0}=t_{11}^{0}$ or $t_{10}^{1}=t_{11}^{1}$

After aligning the recording time bases to adjust the $\mathrm{p}=0$ paths $t_{00}^{i}=t_{01}^{i}$ and the $\mathrm{p}=1$ paths $t_{10}^{i}=t_{11}^{i}$ to be the same for a single interferometer $\mathrm{i}$, the non-interfering terms containing $P_{i n c+c}$ in B7 are expected to be identical. To remove the leading power terms involving $P_{i n c+c}$, we subtract the two amplitude-rescaled and time-shifted recordings in one interferometer and define a single 'signal function' with superscript i for the ith interferometer:

$$
D^{i}=\frac{D_{0}^{i}}{B_{0}^{i}}-\frac{D_{1}^{i}}{B_{1}^{i}}=\frac{1}{P_{c}(0)}\left\{\left[P_{c}\left(t-t_{00}^{i}\right) P_{c}\left(t-t_{10}^{i}\right)\right]^{1 / 2}+\left[P_{c}\left(t-t_{01}^{i}\right) P_{c}\left(t-t_{11}^{i}\right)\right]^{1 / 2}\right\} \cos \theta^{i}
$$

All gains and transmission factors in B8 are replaced by four measured scale factors $B_{d}^{i}$. For the present work, we will include one additional approximation that the delay times for the two interferometers are approximately equal $\tau^{0} \approx \tau^{1}$ (they need not be, but for the present paper they were the same to within 0.8 percent). Under this additional condition where we have aligned the recorder time bases using all $\mathrm{p}=0$ path differences, and also have $\tau^{0} \approx \tau^{1}$, the time delays along the $\mathrm{p}=1$ paths are also equal across both interferometers, $t_{10}^{1}=t_{10}^{0}=t_{11}^{1}=t_{11}^{0}$. The accuracy of this approximation for this paper is within 0.8 percent of $3.2 \mathrm{~ns}$ or to within $25 \mathrm{ps}$. Finally, to eliminate the remaining power dependence in $\mathrm{B} 8$ we form a ratio $\mathrm{R}$ between the two interferometer signal functions $D^{i}$ : 


$$
R=\frac{D^{1}}{D^{0}}=\left(\frac{D_{0}^{1}}{B_{0}^{1}}-\frac{D_{1}^{1}}{B_{1}^{1}}\right) /\left(\frac{D_{0}^{0}}{B_{0}^{0}}-\frac{D_{1}^{0}}{B_{1}^{0}}\right)=\frac{\left[P_{c}\left(t-t_{00}^{1}\right) P_{c}\left(t-t_{10}^{1}\right)\right\rfloor^{1 / 2}+\left[P_{c}\left(t-t_{01}^{1}\right) P_{c}\left(t-t_{11}^{1}\right)\right]^{1 / 2}}{\left[P_{c}\left(t-t_{00}^{0}\right) P_{c}\left(t-t_{10}^{0}\right)\right]^{1 / 2}+\left[P_{c}\left(t-t_{01}^{0}\right) P_{c}\left(t-t_{11}^{0}\right)\right]^{1 / 2}} \frac{\cos \theta^{1}}{\cos \theta^{0}} .
$$

Through timing characterization and time base alignment, we have arranged for times in the numerator and denominator of $\mathrm{B} 9$ to be the same to better than the recording sample interval $\Delta \mathrm{t}$, say, $\Delta \mathrm{t}=100$ or 200 ps. Errors due to rapid power fluctuations are expected to be negligible for fluctuations on time scales as short as the recorder sample interval. For times longer than the sample interval $\Delta \mathrm{t}$, the intensities in the numerator and denominator cancel, and $\mathrm{R}$ greatly simplifies to

$$
R=\frac{D^{1}}{D^{0}} \equiv \frac{\cos \theta^{1}}{\cos \theta^{0}}=\frac{\cos \left(\bar{\omega}_{S}(t-\bar{t}) \tau^{1}+\theta^{1}(0)\right)}{\cos \left(\bar{\omega}_{S}(t-\bar{t}) \tau^{0}+\theta^{0}(0)\right)}
$$

In other words, the incoherent and coherent power fluctuations are eliminated using four detected signals, amplitude characterization, and timing characterization.

The five remaining parameters in expression (B10) are obtained from other characterization measurements: $\tau^{i}$ values that are obtained by calibration of the VPF, $\theta^{i}(0)$ values are determined from the experimental recordings before shock waves arrive, and the total propagation time $\bar{t}$ that can be determined through additional timing characterization. With known characterization parameters, we are now close to obtaining $\bar{\omega}_{s}$ from R.

The expression B10 is a more general case of an expression obtained for other push-pull VISAR systems using polarized optics and a 90 degree interference phase shift imposed between the two polarizations (imposed, in effect, between two interferometers). In other words, with an additional restriction that the VPFs are identically equal, $\tau=\tau^{1}=\tau^{0}$, and by requiring that the interference phase difference is exactly 90 degrees would reduce B10 further to 
$R=\tan \left(\bar{\omega}_{s}(t-\bar{t}) \tau\right)[16,21]$. This is a standard form used in the analysis of push-pull VISARs. An advantage of this form is that it can be inverted analytically using arctangents. Relaxing the requirements that $\tau^{1}=\tau^{0}$ and that the phase difference between the interferometers is 90 degrees requires our analysis to diverge from traditional VISAR methods (we need to solve B10). We relax these constraints somewhat since our interferometer delays are not identical. In the analysis, we allow the VPFs to be different, do not require the phase difference to be 90 degrees, and work with the more general expression in B10 to obtain values for $\bar{\omega}_{S}$. The approach we use to extract $\bar{\omega}_{S}$ is to search numerically for values that satisfy the expression (B10) for each measured value of R at each time t. Solving (B10) directly in the presence of noise creates algorithmic difficulties. Another approach is motivated by noting that the expression in (B10) is satisfied also by a subset (half) of the roots of a different function:

$$
\chi=D^{1} \cos \left(\bar{\omega}_{S}(t-\bar{t}) \tau^{0}+\theta^{0}(0)\right)-D^{0} \cos \left(\bar{\omega}_{S}(t-\bar{t}) \tau^{1}+\theta^{1}(0)\right) \equiv 0
$$

Best values of $\bar{\omega}_{S}$ that most closely satisfy B10 in the presence of noise can be found numerically by searching for the local minima of $\chi^{2}$ from B11.

The expression for $\mathrm{R}$ can be satisfied by many values of $\bar{\omega}_{s}(t-\bar{t})$-- one of these is the correct physical solution. The approach we use for finding the physically correct answer is to first find all possible answers (local minima) up to an expected maximum value for the velocity (i.e., a maximum for the frequency shift $\bar{\omega}_{s}(t-\bar{t})$ ), and to supplement the solutions with other information to decide between the choices. To find all the local minima, the initial phases are found by noting the sign of the phase terms prior to a shot, and then determining the phases from the portion of the data record immediately before breakout. Numerical minimization to obtain a set of solutions for $\bar{\omega}_{s}(t-\bar{t})$ at each time t employs first a trial set of adequately spaced values of $\bar{\omega}_{s}(t-\bar{t})$ between the poles and zeros of B10, choosing preliminary local minima from within the trial sets, and finally employing a combination of steepest descent and Newton's method to refine and converge on a set of best minima for $\chi^{2}$ between the poles in B10. 
Convergence is obtained usually after only a few iterations per minimum. We tested this method using simulated input data including noise and verified that solving for the local minima of $\chi^{2}$ in B11 gives a very good approximation to $\bar{\omega}_{S}$ for a wide range of model parameters (VPFs, signal to noise ratio, initial phases, etc.). Best results are obtained in a configuration similar to traditional VISARs, i.e., equal VPFs and 90 degree starting phase.

Under the VISAR approximation, we assume one physically correct value exists for

$\bar{\omega}_{s}(t-\bar{t})$, i.e., the experiment is designed so that multiple Doppler shifts from different moving interfaces are not observable in the measurement (this is a common restriction placed on the design of experiments employing VISARs). Physical reasoning or comparison to other known data are needed to identify the correct solution. In the case shown in Figs. 6 and 7, the additional information available was the direct observation of the number of oscillations in the raw data corresponding to the number of VPFs in the velocity jump.

\section{REFERENCES}

1. R. W. Armstrong and S. M. Walley, International Materials Reviews 53, 105 (2008).

2. G. I. Kanel', V. E. Fortov, S. V. Razorenov, Physics-Uspekhi 50, 771 (2007).

3. D. Grady, in Shock Compression of Condensed Matter - 2007, edited by M. Elert, M. D. Furnish, R. Chau, N. Holmes, and J. Nguyen (American Institute of Physics, New York, 2007), p. 5.

4. W. J. Nellis, Rep. Prog. Phys. 69, 1479 (2006).

5. B. A. Remington, R. P. Drake, D. D. Ryutov, Rev. of Mod. Phys. 78, 755 (2006).

6. P. M. Celliers, G. W. Collins, L. B. Da Silva, D. M. Gold, and R. Cauble, Appl. Phys. Lett. 73, 1320 (1998).

7. L.M. Barker, in Shock Compression of Condensed Matter - 1999, edited by M.D. Furnish, L.C. Chhabildas, and R.S. Hixson (American Institute of Physics, New York, 1999), p. 11. 
8. A comment is needed on the phrase "any reflector" in use in the acronym VISAR. Clearly, a sufficiently poor target reflector would be unusable for any VISAR. Ranges for the return reflection and power levels demonstrated in this paper are described in the experimental section.

9. L. M. Barker and R. E. Hollenbach, Rev. Sci. Inst. 36, 1617 (1965).

10. P.D. Sargis, N.E. Molau, D. Sweider, M.E. Lowry and O.T. Strand, "Photonic Doppler Velocimetry" Lawrence Livermore National Laboratory document number UCRL-ID-133075 (1999), available at http://www.osti.gov/energycitations/product.biblio.jsp?osti_id=8025

11. O. T. Strand, D. R. Goosman, C. Martinez, T. L. Whitworth, and W. W. Kuhlow, Rev. Sci. Instrum. 77, 083108 (2006).

12. J. Weng, H. Tan, X. Wang, Y. Ma, S. Hu, and X. Wang, Appl. Phys. Lett. 89, 111101 (2006).

13. J. N. Johnson and L. M. Barker, J. Appl. Phys. 40, 4321 (1969).

14. C.F. McMillan, D.R. Goosman, N.L. Parker, L.L. Steinmetz, H.H. Chau, T. Huen, R.K. Whipkey, and S.J. Perry, Rev. Sci. Instrum. 59, 1 (1987).

15. D. R. Goosman, J. Appl. Phys. 46, 3516 (1975).

16. W.F. Hemsing, Rev. Sci. Instrum. 50, 73 (1979).

17. L.M. Barker and R.E. Hollenbach, J. Appl. Phys. 43, 4669 (1972).

18. L. M. Barker and R. E. Hollenbach, J. Appl. Phys. 41, 4208 (1970).

19. W. C. Sweatt, Rev. Sci. Instrum. 63, 2945 (1992).

20. J.H. Nguyen and N. C. Holmes, in Shock Compression of Condensed Matter-2001, edited by M. D. Furnish, N. N. Thadhani, and Y. Horie (American Institute of Physics, New York, 2001), p. 1251.

21. D. H. Dolan, "Foundations of VISAR analysis," Sandia National Laboratory Report SAND2006-1950 available at http://www.osti.gov/bridge/product.biblio.jsp?osti_id=886901.

22. B. J. Jensen, D. B. Holtkamp, P. A. Rigg, and D. H. Dolan, J. Appl. Phys. 101, 013523 (2007).

23. L. Levin, D. Tzach, J. Shamir, Rev. Sci. Instrum. 67, 1434 (1996). 
24. L. Fabiny and A. D. Kersey, IEEE Photon. Technol. Lett. 9, 79 (1997).

25. J. Weng, H. Tan, S. Hu, Y. Ma, and X. Wang, Rev. Sci. Instrum. 76, 093301 (2005).

26. L. M. Barker and K. W. Schuler, J. Appl. Phys. 45, 3692 (1974).

27. D. Hayes, J. Appl. Phys. 89, 6484 (2001).

28. B. M. LaLone, O. V. Fat'yanov, J. R. Asay, and Y. M. Gupta, J. Appl. Phys. 103, 093505 (2008).

29. L. P. Martin, J. R. Patterson, D. Orlikowski, and J. H. Nguyen, J. Appl. Phys. 102, 023507 (2007).

30. S. K. Sheem and T. G. Giallorenzi, Appl. Phys. Lett. 35, 914 (1979).

31. R. J. Clifton, J. Appl. Phys. 41, 5336 (1970).

32. K. Krauter, "Single Mode VISAR," thesis performed at Lawrence Livermore National Laboratory with report number UCRL-TH-236712, accepted by the faculty of California Polytechnic State University at San Luis Obispo towards advancement to an M.S.E.E. degree in Nov. 2007. The thesis is available at http://www.osti.gov/bridge/product.biblio.jsp?query_id=1\&page=0\&osti_id=926025.

\section{FIGURE CAPTIONS}

Figure 1. Single-Mode VISAR design. SMV is composed of two Mach-Zehnder interferometers and four detectors. Fifty percent power splitters are labeled $\mathrm{i}, \mathrm{p}$, and $\mathrm{d}$. Interferometers are labeled $\mathrm{i}=0$, 1; interferometer paths are labeled $\mathrm{p}=0,1$; and each interferometer has two outputs labeled $\mathrm{d}=0$ and 1 . Labels in the figure are: modulator (M); variable attenuator (VA); optical circulator (C); optical power monitor (PM); polarization adjuster (PA); optical delays $(\tau)$; phase shifters (PS) and detectors (D).

Figure 2. SMV calibration performed by measuring the interferometer outputs while changing the optical frequency.

Figure 3. High speed digital recordings of four SMV-interferometer detector outputs obtained during an exploding bridge-wire, foil-flier experiment. 
Figure 4. Velocity results analyzed from SMV recordings obtained from exploding bridge-wire, foil-flier experiments.

Figure 5. Experimental arrangement for direct comparison of PDV and SMV in a gas-gun, impact experiment. The light from a single probe was split and detected using PDV and SMV.

Figure 6. Comparison of mass velocity results analyzed from a gas gun experiment. Both PDV and SMV were used to detect light from a single probe (as depicted in Fig. 5). The upper panel shows two traces, one each for SMV (blue trace) and PDV (red trace). The lower panel shows the percent difference between the traces in the upper panel.

Figure 7. Interferometer fringes observed during an interval corresponding to the interferometer delay time (3.2 ns) after the velocity jump shown in Fig. 6. Two traces are shown for a pair of interferometer detectors exhibiting a relative phase shift of 180 degrees. Dots are the recorder values obtained at sample intervals of $0.2 \mathrm{~ns}$. Lines are drawn between the sampled points as a guide to the eye. 


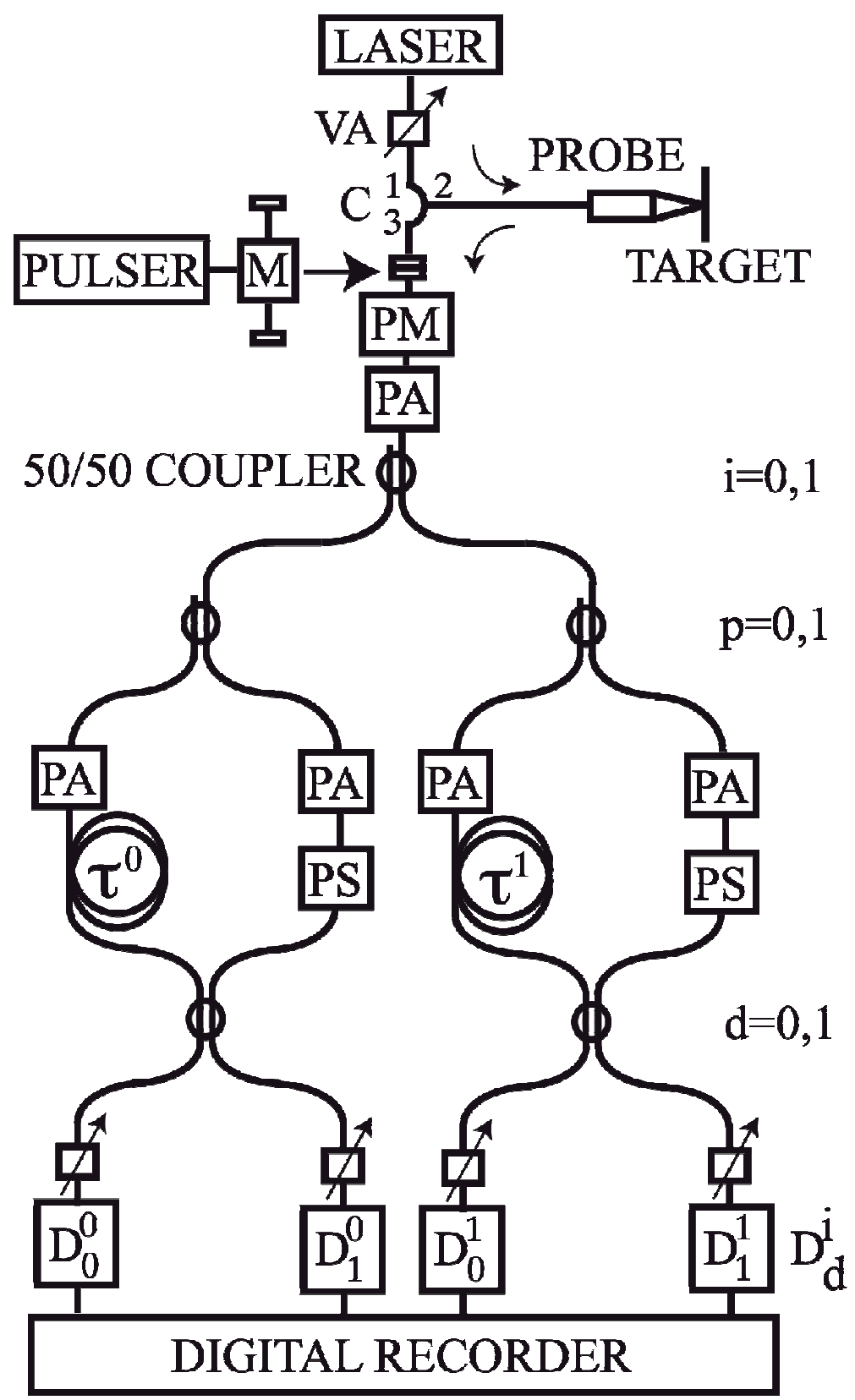

Figure 1. 


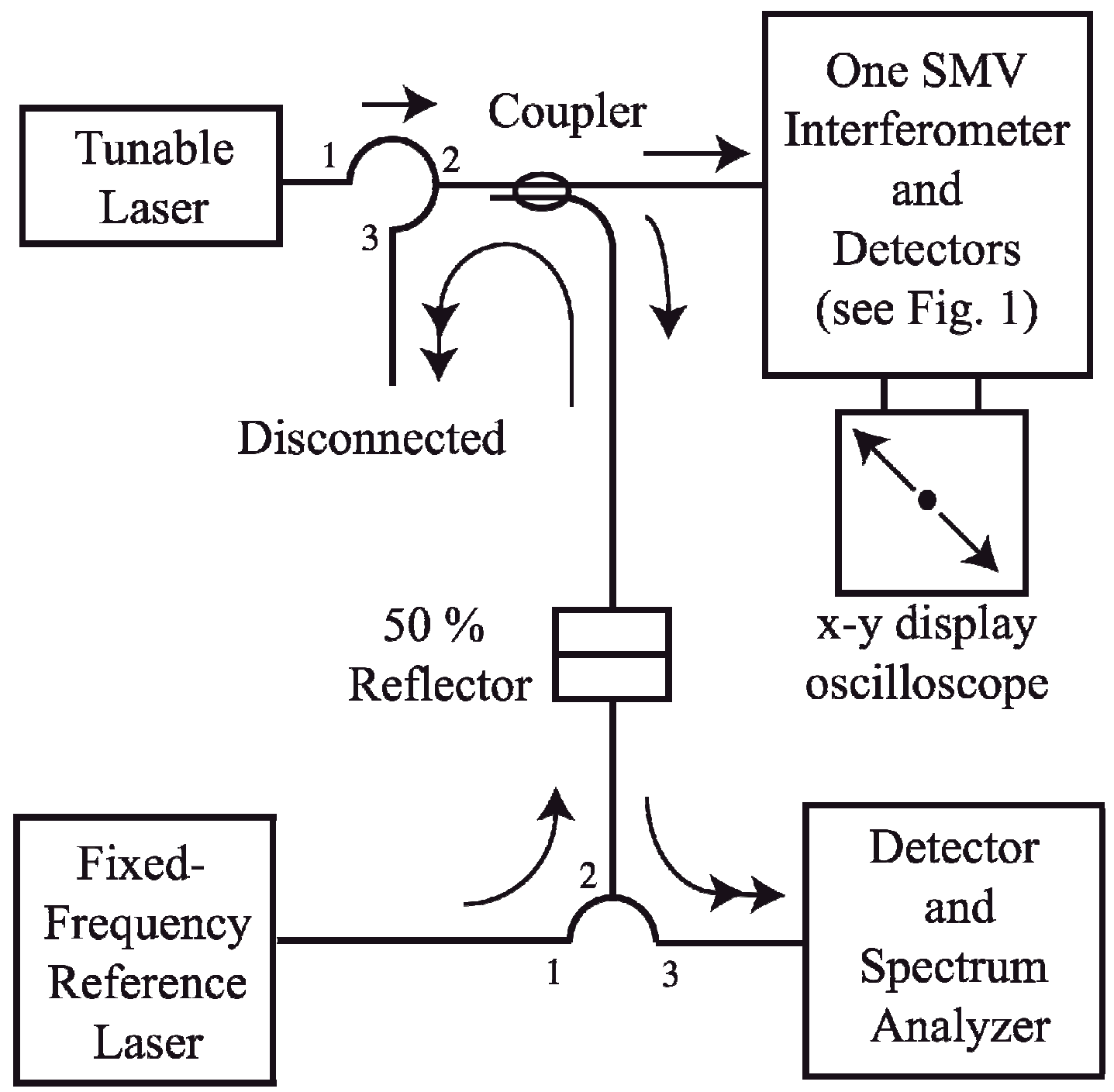

Figure 2. 


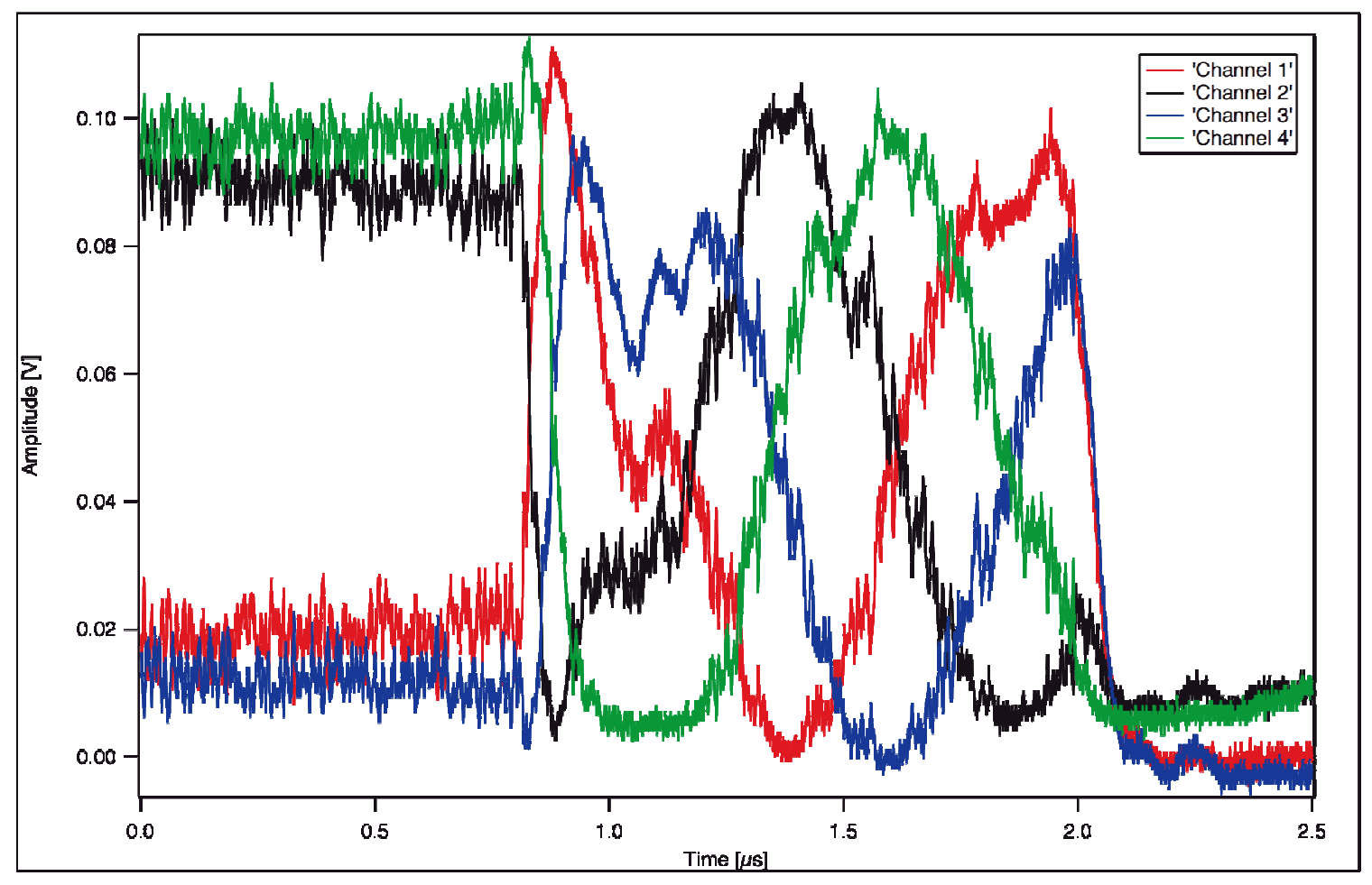

Figure 3. 


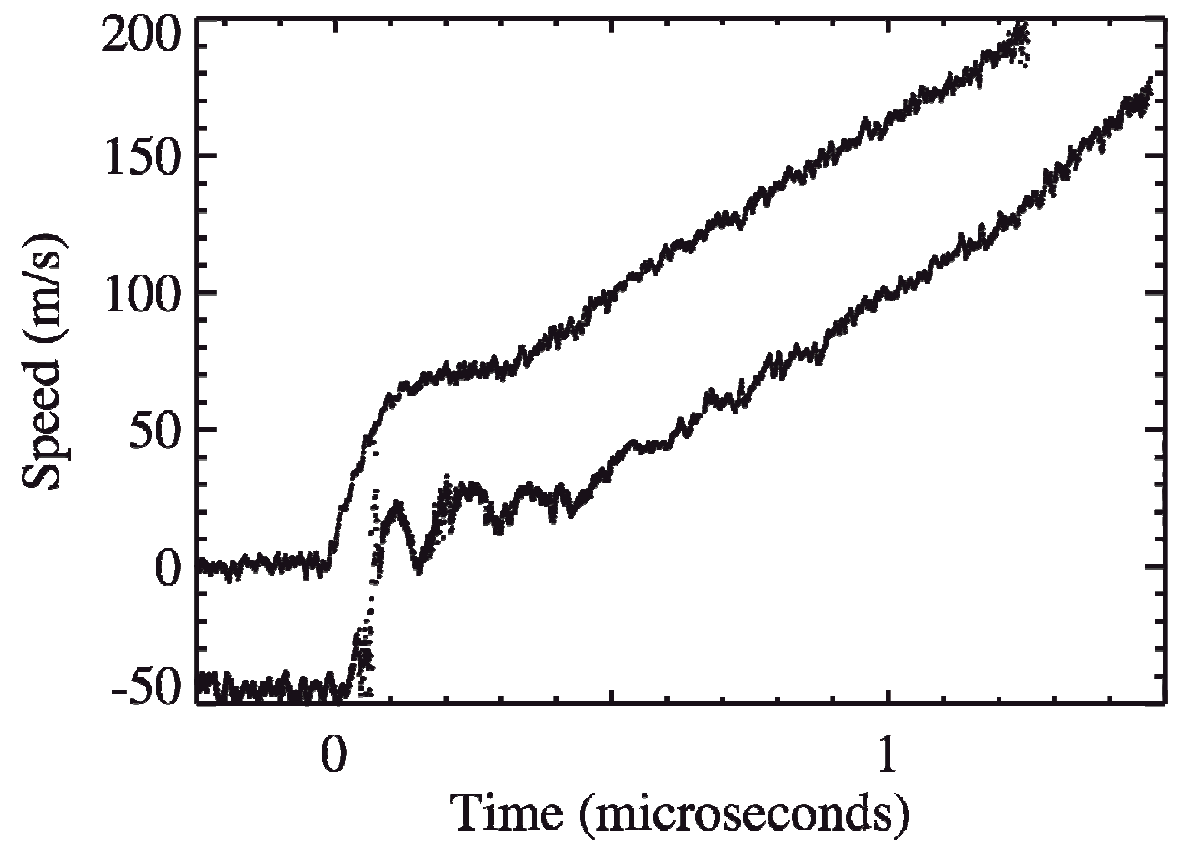

Figure 4. 


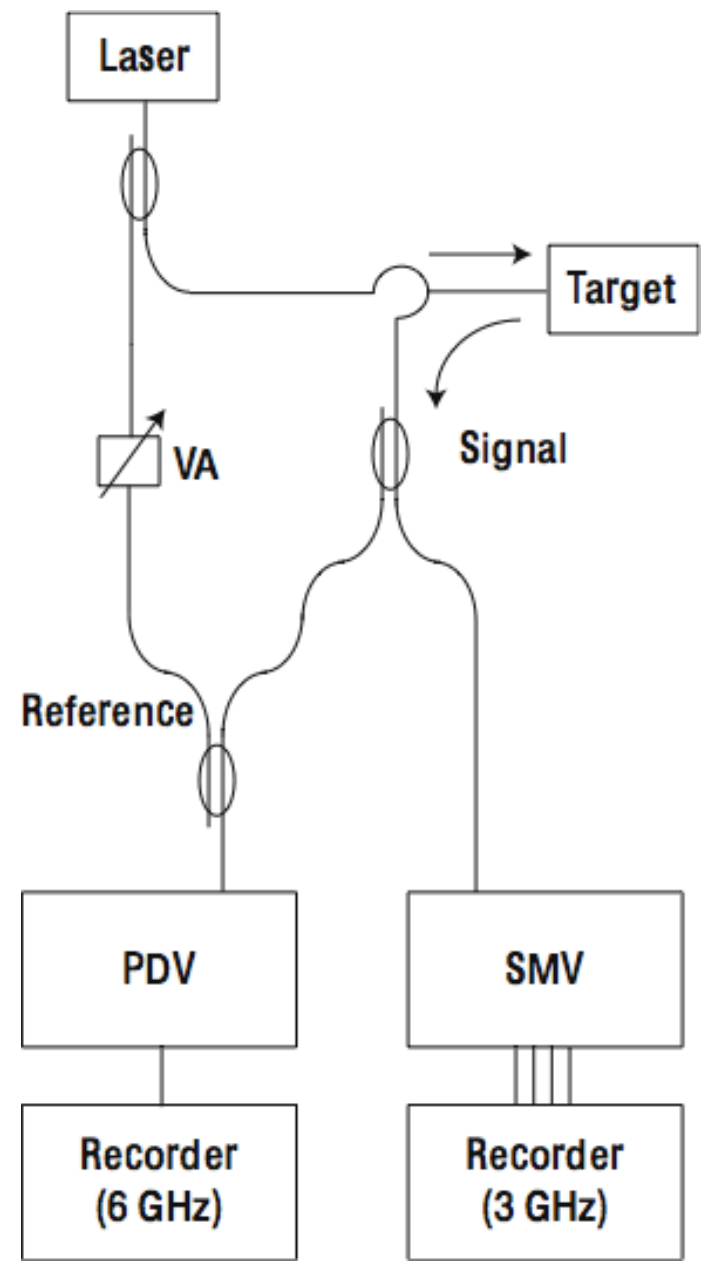

Figure 5 


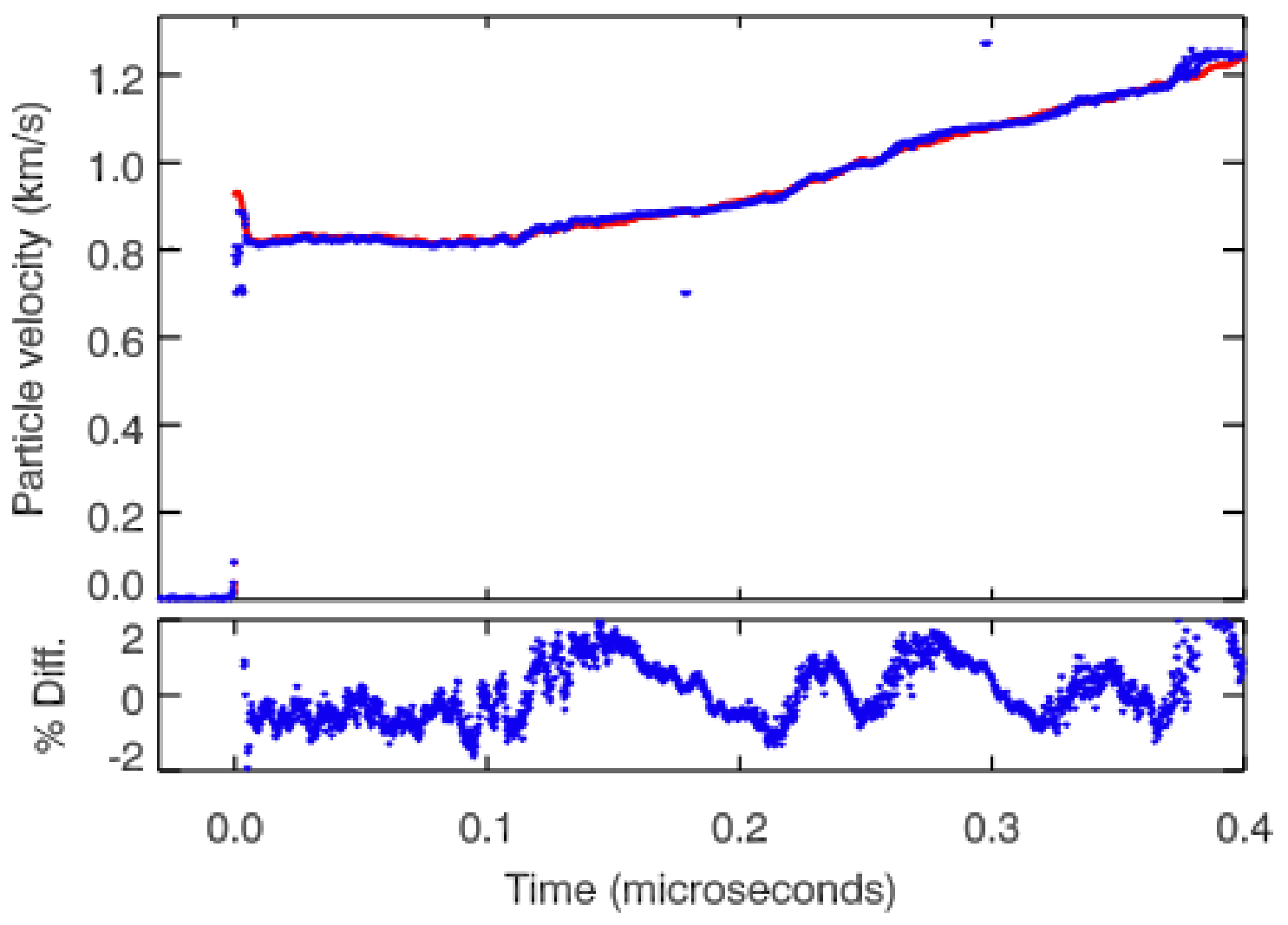

Figure 6 


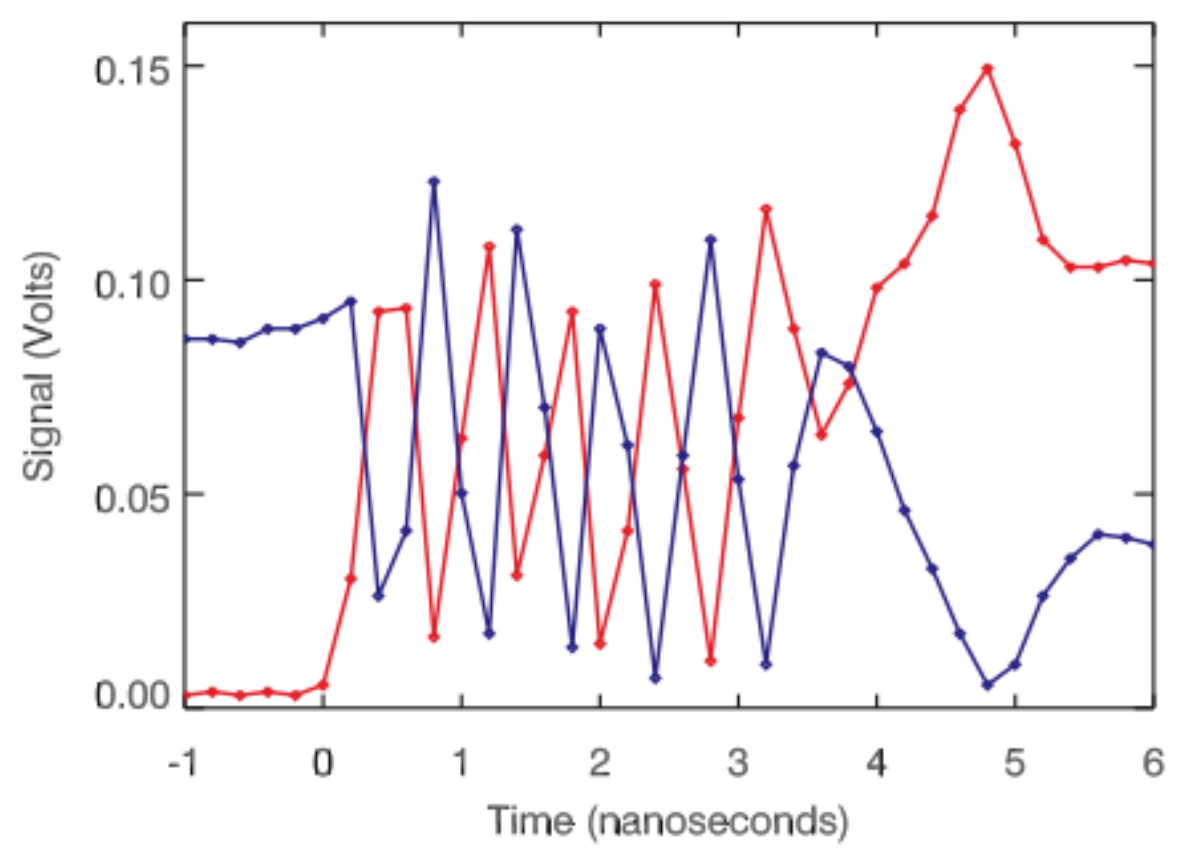

Figure 7. 